\title{
Laboreal
}

Volume $16 \mathrm{~N}^{\circ} 2$ | 2020

Programa de Pesquisa do Curso da Ação

\section{Cognição distribuída e curso de ação}

Cognición distribuida y curso de acción

Cognition distribuée et cours d'action

Distributed cognition and course of action

Jacques Theureau

\section{OpenEdition}

\section{Journals}

Edición electrónica

URL: http://journals.openedition.org/laboreal/16502

DOI: 10.4000/laboreal.16502

ISSN: 1646-5237

\section{Editor}

Universidade do Porto

\section{Referencia electrónica}

Jacques Theureau, «Cognição distribuída e curso de ação », Laboreal [En línea], Volume 16 №2 | 2020, Publicado el 01 diciembre 2020, consultado el 13 diciembre 2020. URL : http://

journals.openedition.org/laboreal/16502 ; DOI : https://doi.org/10.4000/laboreal.16502

Este documento fue generado automáticamente el 13 diciembre 2020.

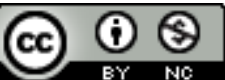

Laboreal está licenciado com uma Licença Creative Commons - Atribuição-NãoComercial 4.0 Internacional. 


\title{
Cognição distribuída e curso de ação
}

\author{
Cognición distribuida y curso de acción \\ Cognition distribuée et cours d'action \\ Distributed cognition and course of action
}

Jacques Theureau

\section{NOTA DEL EDITOR}

Manuscrito recibido en: 02/03/2020

Aceptado tras peritaje en: 26/10/2020

Artigo publicado em língua francesa na revista Activités, vol. 17, n2 de 2020 [Le

programme de recherche cours d'action]

Traducción : Adriana Elena Ramponi [aeramponi@gmail.com]

\section{NOTA DEL AUTOR}

Presento mis agradecimientos a Edwin Hutchins que me dio la oportunidad de iniciar esta reflexión y la estimuló, a Guillaume Azéma, Gilles Dieumegard, Nicolas Donin, Jérôme Guérin, Serge Leblanc, Germain Poizat, Pascal Salembier y Jacques Saury, que ampliaron mi conocimiento de las publicaciones de Edwin Hutchins y sobre todo mi conocimiento acerca de cómo fueron recibidas internacionalmente. Presento también mis agradecimientos a todos aquellos que intercambiaron discusiones en enero de 2019 acerca de una primera versión incompleta de este artículo, y muy particularmente a Guillaume Azéma que me brindó entonces una relectura crítica detallada del mismo, y a Germain Poizat que hizo lo mismo en una segunda y una tercera versión completa, y que se ocupó de las relecturas finales de este artículo. 


\section{Introducción}

1 En ocasión de una conferencia mía sobre su "herencia" (jtítulo impuesto!) (Theureau, 2018), Edwin Hutchins me escribía: “A veces he sentido que debería reunir las ideas que tuve durante los últimos quince años en un documento coherente de la nueva cognición distribuida" (correspondencia personal). Efectivamente, desde la publicación de su obra Cognition in the Wild (Hutchins, 1995), que dio a conocer ampliamente su hipótesis de la 'cognición distribuida', sus colegas y él mismo efectuaron variadas investigaciones que se tradujeron, hace aproximadamente unos doce años, en un cambio importante de perspectiva teórica y metodológica. De esta manera, Edwin Hutchins (2006) volvió sobre esta obra para escribir lo que sigue: "En los años posteriores a su publicación, Cognition in the Wild fue criticada por haber dicho tan poco sobre las personas en situación de navegación. Describe las herramientas del oficio y el desarrollo histórico de dichas herramientas. Describe los procesos sociales y las propiedades cognitivas de esos procesos sociales, pero no dice casi nada sobre las prácticas encarnadas de los navegantes como personas de carne y hueso. En lo esencial, los procesos cognitivos descritos en Cognition in the Wild, y en otros abordajes de la cognición distribuida, se presentan sin referencia al rol del cuerpo en el pensamiento. Es decir que a pesar del hecho de que [la hipótesis de] la cognición distribuida afirma que la interacción de las personas con las cosas es un fenómeno central de la cognición, el planteamiento quedó curiosamente desencarnado" (Hutchins, 2006, p.91, traducción libre). Partiendo de allí, sólo bastaba dar un paso para que Edwin Hutchins comprobara la existencia de un aire de familia entre sus propias investigaciones, la "perspectiva de la enacción" y las diversas investigaciones en términos de "cognición encarnada" que habían comenzado a florecer en esa época (Hutchins, 2010a, 2010b).

2 Sin esperar la "nueva cognición distribuida" prometida por Edwin Hutchins y sin prejuzgar sobre su contenido, apoyándome en más de 30 años de conversaciones periódicas con Edwin Hutchins, de consejos mutuos de lectura de otros autores [1], y de lectura, por mi parte, de sus publicaciones individuales y colectivas y de las de alguno(a)s de sus colaboradore(a)s, voy a exponer aquí los elementos esenciales de lo que podría ser, a mi parecer, un programa de investigación que obtuvo, desde mi punto de vista, el beneficio de los avances realizados por ambos programas de investigación, en parte parecidos, en parte complementarios y en parte alternativos, que fueron durante todos estos años el programa de investigación de la 'cognición distribuida' y el del 'curso de acción' [2]. Un programa de investigación de estas características podría ser llamado "programa de investigación 'curso de acción' aumentado" o, más precisamente pero siempre de manera muy resumida, "programa de investigación sobre la actividad humana como enacción y dinámica cultural". Veremos que, si bien los beneficios en materia de medios heurísticos (herramientas y métodos de construcción de datos y de análisis) de dicho "aumento" del programa de investigación 'curso de acción' (en adelante programa $\mathrm{CdA}$ ) serán considerados secundarios, aquellos en materia de medios teóricos (hipótesis, objetos y nociones teóricas) y de coherencia entre esos medios teóricos serán importantes y podrán dar acceso a nuevas investigaciones empíricas y tecnológicas, a nuevas conexiones entre ellas y a nuevos debates con otros programas de investigación.

3 Además, como para hacerlo tendré que detallar las dinámicas paralelas de ambos programas de investigación 'cognición distribuida' y ‘CdA de 1987 hasta nuestros días, 
un objetivo colateral de este artículo será hacer de este caso real de relaciones entre dos programas de investigación en parte parecidos, en parte complementarios y en parte alternativos, un banco de pruebas para la reflexión epistemológica de cada uno sobre lo que debería ser, idealmente, la relación entre dos programas de investigación de estas características.

4 Si eventualmente mencionaré al pasar hipótesis, nociones, métodos del programa de investigación empírica $\mathrm{CdA}$, así como elementos de su epistemología y de su ética, supondré conocido lo esencial de ello, incluso las nociones de la epistemología de los programas de investigación que desde el comienzo integró este programa, por lo menos a través de los otros artículos de este número, en particular el de Julia San Martín y Germain Poizat, y no los explicitaré. El lector que descubra este programa de investigación o algunas de sus partes y desee profundizar la lectura de este artículo, deberá entonces consultar eventualmente algunas publicaciones, capítulos de libros y artículos publicados con anterioridad. Cuando remita a publicaciones de investigaciones particulares llevadas a cabo en el marco del programa de investigación $\mathrm{CdA}$, será sin apuntar a la exhaustividad, mientras que, con relación a las publicaciones de investigaciones particulares llevadas a cabo en el marco del programa de investigación "cognición distribuida", apuntaré a dicha exhaustividad sin, por ello, pretender alcanzarla. Si bien Edwin Hutchins no habla de programa de investigación tecnológica 'cognición distribuida', mientras que L. Pinsky y yo mismo, pensamos desde el inicio (Pinsky, \& Theureau, 1987) el programa de investigación CdA como articulando dos facetas, una empírica, la otra tecnológica, él y sus colegas dedicaron toda una parte de sus publicaciones a las consecuencias tecnológicas de la hipótesis de la cognición distribuida. No dudaré entonces en ir más allá de los aspectos tecnológicos de estos dos programas de investigación en términos de programa de investigación tecnológico [ $\left.{ }^{3}\right]$.

Desarrollaré el plan siguiente: en las secciones 1 y 2, observaré sucesivamente los desarrollos respectivos de los programas de investigación 'cognición distribuida' y CdA entre 1987 y nuestros días, poniendo el acento en las relaciones entre ellos y a fin de introducir la cuestión que se plantea hoy, de un "programa de investigación CdA aumentado", gracias a la profundización y a la sistematización de los aportes pasados y a la introducción de nuevos aportes del programa de investigación 'cognición distribuida'; en las secciones 3 a 6, presentaré los pocos componentes de ese "programa de investigación $\mathrm{CdA}$ aumentado" que provienen de una organización eventualmente desigual, de los aportes de ambos programas de investigación; por último, en la sección 7, consideraré dos componentes de dicho "programa de investigación CdA aumentado" que corresponden a sus relaciones con otros programas de investigación [ $\left.{ }^{4}\right]$ y que se inspiraron en el único programa de investigación 'cognición distribuida'. Los componentes que especificaré de este "programa de investigación $\mathrm{CdA}$ aumentado" serán entonces, en total, los siguientes: los datos empíricos y la ingeniería de las herramientas de observación, de registro, de transcripción, de cálculo estadístico (sección 3); el estudio sistemático de las relaciones entre las actividades individuales y colectivas que dan lugar o no a experiencia para los actores (sección 4); la ingeniería de las situaciones y la ingeniería de las herramientas cognitivas (sección 5); el análisis de las actividades de investigación científica y tecnológica, actividades de creación artística y, más generalmente, actividades creativas (sección 6); la articulación con las investigaciones sobre la actividad animal como enacción y dinámica cultural y la prosecución del debate con las neurociencias (sección 7). 


\section{Una pequeña historia de la cognición distribuida} investigación. Una propuesta puede constituir un paradigma con dos condiciones: (1) no ser trivial y aportar soluciones a problemas importantes que la ciencia precedente era incapaz de resolver; (2) ser suficientemente abierta para dejarle a los especialistas una gran cantidad de cuestiones a tratar (Kuhn, 1962/1983). Se puede decir que un paradigma es una idea particularmente fecunda en una coyuntura científica, tecnológica y filosófica dada. Un programa de investigación, según su definición por Imre Lakatos [5] (1970), articula: (1) medios teóricos que incluyen eventualmente un paradigma; (2) medios heurísticos fundados teórica y epistemológicamente; (3) cuestionamiento en todo momento de dichos medios teóricos y heurísticos por parte de los hechos empíricos y por otros programas de investigación; (4) evaluación en todo momento del poder heurístico y de la capacidad de crecimiento de dichos medios teóricos y heurísticos.

\subsection{El paradigma y el programa de investigación 'cognición distribuida'}

7 La propuesta de Edwin Hutchins según la cual la cognición está distribuida entre actores y soportes materiales que constituyen juntos un "sistema de cognición distribuida" (o "unidad de ecología cognitiva") constituye obviamente un paradigma. El programa de investigación 'cognición distribuida' articula, por lo menos desde 1987 (cuando tomé conocimiento de ello): (1) ese paradigma acompañado de otros medios teóricos, particularmente del "modelo de flujo de información" que conoció varias y sucesivas formulaciones; (2) el método de "etnografía cognitiva" y la renovación por medio de las tecnologías digitales de las herramientas de observación y de registro del comportamiento y del entorno; (3) la referencia a las matemáticas de los sistemas dinámicos definidos por su estado inicial como potenciales herramientas de modelización sintética; (4) la sumisión al cuestionamiento empírico de descripciones detalladas de procesos cognitivos distribuidos a partir de datos explícitos; (5) la evaluación del poder heurístico y de la capacidad de crecimiento de los medios teóricos y heurísticos detallados anteriormente desde el punto de vista empírico (el conocimiento científico de la cognición humana y animal) y, al mismo tiempo, desde el punto de vista tecnológico (el diseño de herramientas cognitivas y, de manera menos desarrollada, el diseño de capacitación de actores en sistemas de cognición distribuida).

\subsection{Las etapas de desarrollo del programa de investigación "cognición distribuida}

Este programa de investigación 'cognición distribuida' parece haberse desarrollado en tres grandes etapas. Etapa 1: la elaboración del método de etnografía cognitiva en las Islas Trobriand (Papuasia) (Malinovski, 1963, 1985); Etapa 2: la serie de investigaciones en torno de la obra 'Cognition in the wild' (Hutchins, 1995); Etapa 3: el desarrollo de las investigaciones en términos de cognición encarnada y el " $¡$ matrimonio!” (es decir, una alianza oficial, posiblemente fecunda y a largo plazo entre "protagonistas", jen parte

Laboreal, Volume 16 N² | 2020 
parecidos, pero también en parte diferentes) entre los paradigmas de la cognición distribuida y de la enacción.

9 La Etapa 1 dio lugar a la publicación de un libro (Hutchins, 1980) sobre las negociaciones relativas a los intercambios de tierras en las Islas Trobriand y de un capítulo de libro (Hutchins, 1983) sobre la navegación en las mismas Islas Trobriand, retomado luego en 'Cognition in the wild'. El primero apunta a "documentar el tipo de actividades naturales en las que los nativos demuestran sus capacidades de razonamiento (...). Todo este esfuerzo descansó sobre la convicción de que un análisis cuidadoso del comportamiento experto (skilled) en el mundo real puede aportar datos científicos sobre los procesos cognitivos involucrados en la producción de dicho comportamiento" (Hutchins, 1980, p. VII, traducción libre). Cuando lo leí (en 1987), lo que me interesaba era ese aspecto metodológico y la implementación en la modelización del "razonamiento plausible" de George Polya $(1934,1958)[6]$; hoy, lo que me parece muy interesante es la manera cómo este análisis muestra los límites descriptivos y explicativos de la teoría del don, que presumiblemente da cuenta de la economía en esas tierras, y consecuentemente también, la necesidad de transmitir esta teoría del don con análisis de las actividades en juego que me parecen además muy interesantes (ver Theureau, 2019). Pero lo que caracteriza a esta Etapa 1 desde el punto de vista de la construcción del programa de investigación 'cognición distribuida' es, primero, la elaboración del "método de etnografía cognitiva". Tal como lo definió Edwin Hutchins, este método comprende los siguientes elementos: análisis etnográfico de campo poniendo el acento en la dinámica cultural y aumentado por la formación cultural del investigador en las labores practicadas; selección de episodios de actividad; descripción detallada de los procesos cognitivos distribuidos en dichos episodios de actividad en términos de "modelos de flujo de información", por lo tanto, en términos científicamente definidos, a fin de dar cuenta de las dinámicas culturales. Recordemos que el análisis etnográfico clásico (desde Brodislav Malinowski) se basó por el contrario en una concepción estática de la cultura.

10 La Etapa 2 dio lugar a la publicación de la obra 'Cognition in the wild' (Hutchins, 1995), a las principales investigaciones empíricas (simulador de formación en navegación, buque de guerra, cabina de avión, pero también control aéreo, conducción de automóvil, etc.) en relación orgánica con la ingeniería en esos campos, a las investigaciones especulativas sobre el desarrollo del lenguaje (léxico y gramática) (ver secciones 4 y 5) y a una primera serie de investigaciones empíricas sobre la cognición animal que insiste en su carácter colectivo y situado en el entorno (ver sección 7). Lo que caracteriza esta Etapa 2, es la propuesta y la implementación de la noción de "sistema de cognición distribuida" (o "unidad de ecología cognitiva"), que une cognición, colectivo y entorno, así como las de noción analítica de "modelo de flujo de información".

11 La Etapa 3, iniciada aproximadamente en 2005 e inconclusa, vio el desarrollo del programa de investigación 'cognición distribuida' en términos de 'cognición encarnada' y el "matrimonio", que no dio aún todos sus frutos, entre los paradigmas de la cognición distribuida y de la enacción. Lo que era común entre esos paradigmas de la cognición distribuida y de la enacción, y en consecuencia también entre los programas de investigación 'cognición distribuida' y $\mathrm{CdA}$, ya lo era anteriormente: el entorno como interno en la cognición; la cognición como colectiva; la ausencia del "si-mismo" de un actor que se constituiría por fuera del entorno momentáneo de dicho actor, 
incluidos los otros actores en aquel momento. Lo que faltaba explícitamente en la enacción como paradigma pero no en los programas de investigación $\mathrm{CdA}$ y 'cognición distribuida' era: considerar a la tecnología y a la cultura como dinámicas; en el entorno interno de la cognición, la insistencia sobre el entorno técnico; la determinación de una relación orgánica (en diversos grados) entre las investigaciones empíricas y las investigaciones tecnológicas. La introducción de la noción de cognición encarnada y el "matrimonio" entre los dos paradigmas de la cognición distribuida y de la enacción se transformaron para Edwin Hutchins en la precisión de la noción de "práctica cultural" y dieron lugar a investigaciones empíricas sobre la cognición encarnada y distribuida, y a una nueva serie de investigaciones empíricas sobre la cognición animal insistiendo, no sólo en su carácter colectivo y situado en el entorno, como en la Etapa 2, sino también en su carácter encarnado (ver sección 7). La participación activa del programa de investigación 'cognición distribuida' en debates científicos (como parte del poder heurístico de dicho programa de investigación) pasó del debate con el cognitivismo (mantenido también en el programa de investigación CdA y el conjunto de los programas de investigación que tienen como paradigma la enacción), al debate con las pretensiones monopólicas de las neurociencias y, secundariamente, con la corriente llamada de "extended mind" (sobre la cual volveré, sección 7). Lo que caracteriza esencialmente esta Etapa 3, es pues la noción sintética de "práctica cultural", que une cuerpo y cultura. En efecto, una "práctica cultural", es, según Hutchins (2008a), "una práctica tal como existe una ecología cognitiva que la limite o la coordine con las prácticas de otras personas. Esta práctica incluye las formas particulares de ver (oír, emocionarse con, sentir) el mundo"Hutchins, 2008a, p. 2012, traducción libre). A esta definición podemos agregar, sin traicionar a Edwin Hutchins, las maneras particulares de desplazarse, sentarse, dirigirse a otra persona, etc. Una "práctica cultural" de estas características no incluye necesariamente una comunicación simbólica y no da necesariamente lugar a una forma de conciencia en los actores involucrados.

Durante todo este desarrollo, hubo una amplia recepción por parte de la literatura internacional del paradigma 'cognición distribuida', pero con la navegación y la cabina (el cockpit) reducidos a ilustraciones de esta última, sin recepción (o con una recepción reducida) de varios otros componentes del programa de investigación 'cognición distribuida', muy particularmente del método de etnografía cognitiva [ $\left.{ }^{7}\right]$. Como vamos a verlo ahora, la recepción por parte del programa de investigación CdA de los trabajos de Edwin Hutchins y de sus colaboradores/as, tal como la percibo hoy, se efectuó gradualmente, interesándose en los diferentes tipos de situaciones estudiadas por ellas mismas y considerando de manera eventualmente crítica todos los componentes del programa de investigación 'cognición distribuida' y no sólo su paradigma.

\section{Y durante aquel tiempo, el programa de investigación CdA...}

13 En lo que respecta al programa de investigación CdA, insistiré en tres puntos: (1) las relaciones que éste mantuvo con el programa de investigación 'cognición distribuida', globalmente entre 1987 y 1996 y, luego, de manera puntual; (2) las principales inflexiones [8] independientes de esas mismas relaciones que dicho programa experimentó desde 1987 hasta nuestros días; (3) los niveles de análisis de las actividades humanas que son comunes a ambos programas de investigación y los que no los 
son(insistiendo en las consecuencias metodológicas de estos últimos). Concluiré esta sección de la manera en que actualmente se presentan la cuestión de un "programa de investigación “curso de acción' aumentado" y su respuesta.

\subsection{Los aportes del programa de investigación 'cognición distribuida' al programa de investigación 'CdA desde su primera expresión (1987)}

Hace más de 30 años, en 1987, durante aquel primer encuentro con Edwin Hutchins en San Diego, Leonardo Pinsky y yo (Pinsky \& Theureau, 1987) [ $\left.{ }^{9}\right]$ acabábamos de realizar la constatación de un aire de familia entre la "perspectiva de la enacción" y nuestras investigaciones pasadas, misma constatación que hizo más recientemente Edwin Hutchins entre esta perspectiva y sus trabajos. Mis conversaciones y las de Leonardo Pinsky entre 1986 y 1990 con Donald Norman, Edwin Hutchins, Aaron Cicourel y Michael Cole en San Diego, con Sylvia Scribner en Nueva York y con Jon Barwise, John Perry, Jean Lave y Lucy Suchman en Palo-Alto, nos condujeron a dejar la referencia a la psicología universitaria francesa de esa época en favor de la referecia a la antropología cognitiva, es decir, al desarrollo de la antropología cultural hacia el tema de la cognición (más precisamente, al estudio de las actividades cognitivas cultural y situacionalmente específicas), de la exigencia de modelización, de la articulación con la ingeniería. Esto permitió dejar atrás reacciones pasionales e improductivas suscitadas por nuestros trabajos en favor de debates estimulantes, pagando un alto costo en dificultades institucionales.

Por el contrario, la integración, mediando transformación, de la totalidad del método de etnografía cognitiva, llevó más tiempo. Leonardo Pinsky y yo, tendríamos que haber integrado inmediatamente las focalizaciones selectivas sobre episodios de actividad (actividad concebida, sin embargo, como individual-colectiva y colectiva-individual y no sólo colectiva como en Edwin Hutchins) en el análisis de dichos episodios en términos científicamente definidos (y no en términos de "flujo de información", como en Edwin Hutchins) que ya practicábamos, y en la definición dinámica de la cultura que participaba en esta etnografía cognitiva. Sin embargo, fue sólo en mis cursos en la Universidad de Tecnología de Compiègne en 1997 [10], que propuse dar todo su lugar al análisis etnográfico clásico revisado, poniendo el acento en las dinámicas culturales (Theureau, 2006). Inicialmente pensábamos, muy pragmáticamente y teniendo en cuenta la eficiencia de nuestros métodos de construcción de datos - los que, enriquecidos y repensados participaron en lo que llamé más tarde "el observatorio del programa de investigación CdA" -, que un sondeo económico, social, cultural y político rápido combinado con la construcción, junto con los actores, de métodos concretos de recogida de datos y de análisis a implementar con su colaboración, bastaba para obtener resultados empíricos y tecnológicos [11]. Sin embargo, cabe señalar que cuando se efectuó esta integración del método de etnografía cognitiva en el programa de investigación $\mathrm{CdA}$, fue acompañado de un primer enriquecimiento que consideró el interés de este programa e los diferentes horizontes temporales de las actividades humanas: en vez de focalizaciones selectivas aisladas sometidas a análisis locales, se trató de unir "en historias" dichos análisis locales de focalizaciones selectivas, a través de diversas nociones sintéticas. Señalemos por último que esta integración del método de etnografía cognitiva se tradujo de manera no sistemática en investigaciones 
particulares posteriores. A mi entender, la investigación de Dufresne (2001) sobre el control del tráfico ferroviario en Canadá $\left.{ }^{12}\right]$, que dio lugar sólo a publicaciones ergonómicas prácticas, fue la única de estas investigaciones que implemetó sistemáticamente las herramientas clásicas perfeccionadas por Brodislav Malinowski (las notas de campo y el diario de etnografía) durante un período de tiempo importante in situ. En otras investigaciones particulares, por ejemplo aquellas sobre las actividades de composición musical que realicé en colaboración con Nicolas Donin, se puso mucho más atención en la apropiación cultural previa del medio musical considerado por parte de los investigadores.

Por último, como lo escribí antes, el programa de investigación CdA no dio el monopolio al análisis de la actividad colectiva y la definición que allí fue dada de los objetos teóricos de estudio de la actividad colectiva se inspiró ampliamente en la hipótesis de la cognición distribuida. Si bien hemos hablado de "articulación colectiva de los cursos de acción individuales-sociales, o individuales-colectivos" [13] , y no de "sistema de cognición distribuida" o de "unidad de ecología cognitiva" como lo hacía Edwin Hutchins, hemos detallado en efecto con fórmulas diversas que esta articulación colectiva involucraba "a actores dotados de su entorno intersticial y sumergidos en un entorno más amplio", atribuyendo así, tras Edwin Hutchins, un rol fundamental en la actividad colectiva a la combinación de ese entorno intersticial con ese entorno más amplio.

17 Podemos agregar que las conversaciones en San Diego y en París, durante todos esos años, y las críticas hechas por Edwin Hutchins a nuestros métodos de recogida de datos verbales entre actores $\left.{ }^{[14}\right]$ y a algunos de mis ensayos teóricos provisorios inspirados en la semiótica especulativa peirceana $\left.{ }^{[15}\right]$ contribuyeron al desarrollo del programa de investigación CdA. Muy recientemente, durante la preparación de la conferencia citada más arriba sobre la "herencia de Edwin Hutchins", la lectura más atenta de Hutchins y Johnson (2010), de la que hablaré luego (sección 7), y de Hutchins (2012), me hizo descubrir el interés de la noción de "práctica cultural" allí desarrollada. Veremos (sección 4) que esta noción de "práctica cultural", una vez introducida en el programa de investigación $\mathrm{CdA}$, puede verse enriquecida gracias a nociones desarrolladas independientemente en el marco de este programa y contribuir así a explicar la génesis de un "sistema de cognición distribuida" (o "unidad de ecología cognitiva").

\subsection{Las grandes inflexiones del desarrollo del programa de investigación CdA independientes de los aportes del programa de investigación 'cognición distribuida'}

18 El programa de investigación CdA, después de su primera expresión en 1987 (ver más arriba), experimentó varias inflexiones teóricas y metodológicas, independientes de los aportes del programa de investigación 'cognición distribuida' que acabo de mencionar. Estas últimas surgieron ya sea de las dificultades encontradas en las investigaciones empíricas particulares, ya sea de confrontaciones con investigaciones empíricas llevadas a cabo por otros autores, o bien, de problemas de coherencia teórica entre nociones tomadas de otros autores que sufrieron una transformación insuficiente. observemos sin embargo que, cuando incluyen un desarrollo en relación con la etnografía cognitiva o con la actividad colectiva, estas inflexiones independientes remiten en parte al programa de investigación 'cognición distribuida'. 
Podemos distinguir las inflexiones independientes siguientes:

- Inflexión independiente 1: sistematización en Theureau (1990, 1992), Pinsky (1992), y Theureau y Jeffroy (1994) de los avances logrados desde 1987, que constituyó lo que llamé más tarde el "método elemental" (Theureau, 2004): el análisis empírico limitado de las actividades humanas de trabajo y de uso de productos y la ingeniería ergonómica asociada (artefactos, organización, formación);

- Inflexión independiente 2 (entre 1995-1996 y 2000): desarrollo de las investigaciones sobre las actividades deportivas seguido de las actividades en educación (formadores, formados, articulación colectiva de sus actividades), que dio paso esencialmente a: (1) una exploración más desarrollada de las actividades colectivas, incluso agonísticas, y de la construcción de saberes a través de la actividad humana; (2) un refinamiento por parte de los actores en la expresión de sus sensaciones y emociones durante el desarrollo de su actividad, (3) un traspaso de la ingeniería ergonómica hacia la ingeniería de las situaciones de formación, educación y entrenamiento y una apertura a la ingeniería de las situaciones en general;

- Inflexión independiente 3 (1997-2006): la primera expresión, luego la construcción sistemática de lo que llamé el "método desarrollado" (Theureau, 2006), que integraba explícitamente el método de etnografía cognitiva y proponía una noción de signo hexádico, con seis componentes, modelizando la enacción en un instante dado, y más precisamente traduciendo esta última en un conjunto organizado de seis categorías de fenómenos documentables, lo que permitía un análisis sistemático de la construcción de los saberes en la actividad y no sólo de su implementación

- Inflexión independiente 4 (desde 2003): desarrollo de las investigaciones deportivas en términos de 'curso de in-formación' y de articulación colectiva de los 'cursos de información' dentro de los límites de los datos disponibles sobre los comportamientos, los parámetros fisiológicos y los procesos materiales;

- Inflexión independiente 5 (también desde 2003): las investigaciones sobre las actividades musicales (de las cuales algunas se asociaron a investigaciones en ingeniería cultural) que dieron paso, de manera más general, a investigaciones sobre las actividades creativas a largo plazo en todo tipo de campos socio-técnicos;

- Inflexión independiente 6 (más recientemente): desarrollo de las primeras investigaciones en términos de análisis de actividades sobre varios niveles en relaciones de par estrella (en adelante "par*") [16];

- Serie de inflexiones independientes asociadas al desarrollo de la noción de apropiación que interfieren en ciertas inflexiones independientes anteriores, y marcan las tres etapas de una construcción: (1) la apropiación como desplazamiento de la frontera actor-entorno que se ubica en el centro de la ción de enacción; (2) los tres tipos de apropiaciones (in-situación, in-corporación, in-culturación) en relación con la actividad individual-colectiva de un actor; (3) la apropiación organizacional $\left[{ }^{17}\right]$ y sus diferentes tipos (apropiación institucional o convencional, apropiación-acción organizacional, apropiacióntransformación organizacional $\left.{ }^{[18}\right]$ ) en relación con la articulación colectiva de las actividades individuales-colectivas de varios actores con su entorno intersticial, sumergidos en un entorno más amplio. 


\subsection{Los programas de investigación CdA y 'cognición distribuida': una comunidad de niveles de análisis de las actividades humanas hasta el proyecto reciente de análisis multiniveles de las actividades humanas}

Los objetos teóricos (u objetos de conocimiento) que se estudian habitualmente en e marco del programa de investigación CdA corresponden a lo que podemos llamar los niveles medios de análisis de las actividades humanas, si se considera: (1) que los niveles inferiores de análisis de las actividades humanas vinculan una descripción de las dinámicas neuronales de un actor a elementos del entorno de dicho actor, como en el programa de investigación en neuro-fenomenología iniciado por F. Varela (ver, por ejemplo, Varela, 2017); (2) que los niveles medios de análisis de las actividades humanas corresponden a las actividades individuales-colectivas de actores individuales $o$ a las actividades colectivas-individuales de pequeños colectivos de actores (a menos que se reduzcan conjuntos de actores a actores colectivos) dentro de los límites temporales, espaciales y organizacionales estrechos (por ejemplo, la duración máxima de actividad que personalmente tuve en cuenta hasta hoy en una investigación referida a una actividad individual-colectiva fue de cinco años); (3) que los niveles superiores de análisis de las actividades humanas amplían a diferentes grados dichos límites temporales, espaciales y organizacionales de los colectivos y de sus entornos intersticiales y dan paso así al estudio de las dinámicas organizacionales, sociales, históricas, políticas y culturales.

21 El programa de investigación 'cognición distribuida', por su parte, si bien no habla de niveles de análisis de las actividades humanas, si lo hace de niveles de estudio de la cognición humana. Las investigaciones particulares llevadas a cabo hasta hoy abarcan en parte (puesto que excluyen el estudio de las actividades individuales-colectivas) los niveles medios de análisis de las actividades humanas que acabo de precisar pero ampliando dichos niveles medios de análisis a las actividades animales.

Sin embargo, en el marco del programa de investigación 'curso de acción', el proyecto reciente de un análisis de la actividad humana sobre varios niveles en relaciones de par* (la inflexión independiente 6 indicada en párrafos anteriores) retira este programa de investigación de esta comunidad de niveles de análisis de la actividad humana. En este análisis multiniveles: los niveles relativamente inferiores son los niveles medios de los que acabamos de hablar; los niveles relativamente superiores corresponden al conjunto de las actividades a diferentes escalas temporales, espaciales, organizacionales y culturales, por ejemplo una empresa, una región, etc. Este proyecto fue presentado especulativamente en Theureau $(2015,2019)$. En parte en forma independient y en relación con concepciones de la etnografía cultural eventualmente más específicas, este análisis fue también más o menos implementado empíricamente de diversas formas por otros investigadores, por ejemplo por San Martin (2015), Perrin y Vanini de Carlo (2016) y Watteau (2017). El proyecto vuelve a tomar a la etnografía cultural de campo más o menos clásica como base de una fenomenología de la actividad humana en los niveles superiores, después de haberlo orientado hacia el conocimiento de las dinámicas culturales y haber superado las barreras entre etnografía cultural, sociología, historia y hasta economía política. Este análisis de la actividad humana a varios niveles de las relaciones de par* no fue contemplado por Edwin Hutchins; fue su colega y amigo Aaron Cicourel quien había planteado premisas del mismo.

Laboreal, Volume 16 N² | 2020 
Hemos visto antes que la integración del método de etnografía cognitiva en el programa de investigación CdA fue acompañada de una primera transformación, la puesta en relación del análisis conjunto de varios períodos sucesivos-desfasados seleccionados de la actividad. Este análisis de la actividad humana a varios niveles de las relaciones de par* implica una nueva transformación del método de etnografía cognitiva que, contrariamente a ella y a su primera transformación en el marco del programa de investigación $\mathrm{CdA}$, modifica la etnografía cultural de campo clásica. En lugar de las nociones estáticas de esta etnografía cultural de campo clásica, tenemos; una fenomenología empírica a partir de los datos de etnografía cultural cuyas categorías descriptivas son lo que se puede llamar "tendencias sintéticas de actividades" variadas de diversos conjuntos de actores [19]; una relación de par* entre esta última y la fenomenología empírica de las actividades que dan lugar a conciencia prereflexiva (o experiencia) a partir de los datos sobre los períodos seleccionados de la actividad, es decir las categorías descriptivas de los niveles medios de dichas actividades que dan lugar a conciencia prerreflexiva (o experiencia).

\subsection{La cuestión que se plantea hoy es pasar de una serie de aportes del programa de investigación 'cognición distribuida' efectuados al programa de investigación $\mathrm{CdA}$, a la formulación de un programa CdA aumentado.}

En suma, si encontramos en mis publicaciones, como en las de Leonardo Pinsky o en la de otros autores que desarrollan este programa de investigación CdA referencias positivas a las publicaciones en términos de 'cognición distribuida', estas van acompañadas en general de críticas y limitaciones - hablé, por ejemplo, respecto de la hipótesis de la 'cognición distribuida', de "verdad [aspecto positivo] limitada [aspecto crítico] del colectivismo metodológico" (Theureau, 2006, p. 87, traducción libre) y de “inspiración por la "cognición socialmente distribuida" y sus límites" (ibídem, p. 116, traducción libre).

El punto de conflicto entre ambos programas de investigación residía de hecho tanto en la divergencia entre el paradigma de la cognición distribuida y el de la enacción como en la traducción de esta divergencia en los datos recabados y en la teoría que rige el análisis de estos últimos, ya que el "modelo del flujo de información" describe el "sistema de cognición distribuida" (o "unidad de ecología cognitiva") por un lado, y por otro la "teoría de la actividad-signo" describe el "curso de acción" (o concatenación de instantes de enacción que dan lugar a conciencia prerreflexiva o experiencia). Más específicamente, en el programa de investigación 'cognición distribuida', el conjunto organizado de las categorías descriptivas de los fenómenos se refiere a los fenómenos de la cognición distribuida tales como aparecen sólo al observador científico - sea esto el resultado o no de una interacción más o menos valiosa con los actores y de una invitación a proporcionar su punto de vista espistemológico - y se limita al "modelo de flujo de información". La justificación de su implementación se efectúa a partir de datos de comportamiento de los actores y de datos sobre los procesos materiales. En el programa de investigación $\mathrm{CdA}$, el conjunto organizado de las categorías descriptivas de los fenómenos está constituido por la "teoría de la actividad-signo" y se refiere a los fenómenos de la actividad como enacción de un actor determinado al que se le presentan a través de su conciencia prerreflexiva, y que el observador-interlocutor 
científico puede documentar gracias a los diferentes métodos de acceso a esa conciencia prerreflexiva. La justificación de su implementación se efectúa a partir de tales datos de conciencia prerreflexiva y de datos suplementarios, tanto sobre los comportamientos de los actores como sobre los procesos materiales en juego.

Si esta divergencia teórica no impide (y no ha impedido) la discusión científica a partir de los datos construidos por uno $\mathrm{u}$ otro de estos dos programas de investigación, es porque al mismo tiempo, por objetivos heurísticos (de sumisión más amplia a datos empíricos y de debate con otros programas de investigación) y de contribución a la ingeniería de las situaciones (por ejemplo, la comparación entre opciones de concepción alternativas de las situaciones), los estudios e investigaciones sobre los cursos de acción y su articulación colectiva desembocan en general, en un segundo tiempo - o sea después de la realización del primero - en tesis sintéticas de tipo: "en esta actividad humana, todo ocurre como si... en tales o cuales condiciones". Este "como si..." puede ser expresado en términos objetivistas del mismo tipo que los del "modelo de flujo de información" (o "como si la cognición distribuida consistiera en desplazamientos y transformaciones de especificaciones de una entidad llamada 'información"'). Si bien resulta imposible explicitar y comparar aquí ciertos análisis en términos de "flujo de información" y de "actividad-signo" que hubieran sido efectuados a partir de actividades idénticas o parecidas en una situación idéntica o parecida, visto que tales investigaciones comunes no sucedieron, daré (en la sección 4) un panorama de lo que podrían ser esa explicitación y esa comparación a partir de una investigación empírica particular llevada a cabo en términos de curso de acción.

Una vez convocado en paradigma de la enacción por el programa de investigación 'cognición distribuida', aunque Edwin Hutchins no haya establecido aún todas las consecuencias, y cumplidos los diversos avances de ambos programas de investigación CdA y 'cognición distribuida' (que acabo de resumir en estas dos primeras secciones), la cuestión que se plantea hoy es la de la integración sistemática de nuevos aportes del programa de investigación 'cognición distribuida' al programa de investigación CdA. Esto daría origen, como lo escribí en Introducción, a un "programa de investigación 'curso de acción' aumentado", o, más precisamente, "un programa de investigación sobre la actividad humana como enacción y dinámica cultural", que estaría "en relación orgánica con uno o varios programas de investigación tecnológica y en relación orgánica con uno o varios programas de investigación filosófica".

En las secciones siguientes, voy a precisar algunos componentes esenciales de este "programa de investigación 'curso de acción' aumentada" o "programa de investigación sobre la actividad humana como enacción y dinámica cultural". Pero previamente es conveniente subrayar también, que la integración de los conocimientos del programa de investigación 'cognición distribuida' en este programa de investigación, no estará completa sino mediando, en ciertos casos, una conceptualización original. Es lo que muestra, por ejemplo, una investigación reciente (Dieumegard \& Nogry, 2018) que hace a su vez su contribución al programa de investigación CdA y se inspira en parte, desde una perspectiva de diseño de herramientas cognitivas para la formación y la educación, en una investigación de Hutchins (2005) $\left[{ }^{20}\right]$. 


\section{El observatorio y el taller. los datos empíricos y la ingeniería de las herramientas de observación, de registro, de transcripción, de cálculo estadístico.}

Habiendo anunciado en párrafos anteriores que los beneficios en materia de medios heurísticos de este programa de investigación 'curso de acción' aumentado son secundarios, comenzaré por exponer estos últimos. Subrayemos, sin embargo, que la ilusión de la independencia total de los métodos (que surgen en el "observatorio" o en el "taller", para retomar las nociones desarrolladas en el marco de la epistemología general enactiva y que valen evidentemente para los programas de investigación sobre las actividades humana $\left[{ }^{21}\right]$ ) en comparación con las teorías, corre el riesgo de acrecentarse en cuanto nos apoyamos en el desarrollo técnico para renovar dichos métodos. Como es el caso en los programas de investigación 'cognición distribuida' y 'curso de acción', el enriquecimiento del observatorio y del taller del segundo por los del primero debe ser efectuado prestando particularmente atención a su coherencia con las teorías: (1) coherencia del observatorio resultante con los objetos teóricos; (2) coherencia del taller resultante con los objetos teóricos y las teorías [22] al mismo tiempo.

Entre las condiciones de posibilidad de un "programa de investigación 'curso de acción' aumentado" ya reunidas y que perduran, se encuentra la compatibilidad entre los datos recabados en el marco de ambos programas de investigación. De esta manera, las herramientas y métodos del observatorio de los cursos de experiencia, de los cursos de acción y de los cursos de in-formación y de sus articulaciones colectivas desarrollados en el marco del programa de investigación CdA y las herramientas y métodos de colecta de datos desarrollados en el marco del programa de investigación 'cognición distribuida' pueden acumularse. Podemos entonces retomar el DEW (Digital ethnographer workbench) Manifesto lanzado por Edwin Hutchins ampliando su contenido a fin de considerar la conciencia prerreflexiva o experiencia de los actores. En un discurso plenario en la Cognitive Science Society, Hutchins (2008) precisaba esta etnografía digital como: (1) la extensión de los horizontes de la ciencia cognitiva focalizándose en los fenómenos susceptibles de ser los más interesantes: la actividad cognitiva en el mundo real; (2) la comprensión de lo que son dichos fenómenos y de lo que debemos hacer para mejorar esa comprensión; (3) el desarrollo de teorías que explican lo que se produce en esas actividades entre el cerebro, el cuerpo y el mundo y lo que nos permite predecirlo; (4) la adaptación y el desarrollo de herramientas y métodos apropiados a los desafíos de la puesta a prueba empírica de conjeturas teóricas sobre la cognición en el mundo real, que constituye la misión de la plataforma de etnografía digital (Dew); (5) la difusión de la nueva concepción de la ciencia cognitiva a los etnógrafos cognitivos y a la comunidad científica más amplia (“"armándose de paciencia”, agregaba!).

Esta plataforma de etnografía digital (Dew) suma observatorio y taller. Citemos, entre las publicaciones asociadas: Fouse y Hollan (2010); Fouse, Weibel, Hutchins y Hollan (2011); Weibel, Fouse, Hutchins y Holla (2011); Weibel, Fouse, Emmenegger, Friedmann, Hutchins y Hollan (2012). Esta renovación vale para la cabina (cockpit) (ver Hutchins, Weibel, Emmenegger, Fouse, \& Holder, 2013), pero también para los animales en su entorno (ver, por ejemplo, una investigación sobre las vocalizaciones de los delfines en cautiverio: Johnson (2015) y Karnowski, Hutchins y Johnson (2015)). 
32 En cuanto al taller propiamente dicho, los esfuerzos fueron más modestos en lo referido al programa de investigación $\mathrm{CdA}$, pero no por ello inexistentes. Si, en materia de observación y de registro, se trató sobre todo de adaptar los usos de herramientas desarrolladas (por ejemplo, diferentes tipos de cámaras de video), encontramos también esfuerzos, por ejemplo, en materia de pre-análisis, de cálculo estadístico y de análisis (Perrin, Theureau, Menu, \& Durand, 2011; Goldszmidt, Donin, \& Theureau, 2007). Evidentemente, como estos datos no se limitan a los de observación y registro sobre los cuerpos y el entorno sino que le agregan datos a la conciencia prerreflexiva (o experiencia) de los actores, que son externos al programa de investigación 'cognición distribuida', los aportes de renovación de las herramientas de observación y de registro sobre los cuerpos y el entorno, y de transcripción y de análisis estadístico efectuado en el marco de este programa de investigación 'cognición distribuida' deben ser, por su parte, mejorados.

\section{La apertura de un estudio sistemático de las relaciones entre las actividades individuales y colectivas que dan lugar y no dan lugar a experiencia para los actores.}

Consideraré en esta sección tres series de fenómenos con respecto a las "actividades colectivas-individuales", tal como las considera el programa de investigación CdA, versus un "sistema de cognición distribuida", tal como lo considera el programa de investigación 'cognición distribuida'.

La primera serie es la de los fenómenos de des-colectivización de las actividades colectivas sobre la cual el programa de investigación CdA insistió desde el principio a partir de las nociones de actividad individual-colectiva y de actividad colectivaindividual. La segunda serie es la de los fenómenos de construcción de un "sistema de cognición distribuida" (o "unidad de ecología cognitiva") a partir de la articulación colectiva de las actividades individuales-colectivas de los actores que terminan por componerlo/a.

Estas dos primeras series de fenómenos enriquecen los fenómenos considerados hasta hoy por el programa de investigación 'cognición distribuida". Si la primera pudo ser formulada desde el principio y puesta a prueba en investigaciones empíricas, la segunda, por su parte, tuvo que esperar para poder ser precisada como voy a hacerlo en las próximas páginas de manera todavía puramente especulativa, esperando su puesta a prueba empírica: por el lado del programa de investigación 'cognición distribuida', la integración del paradigma de la enacción y la precisión de la noción sintética de "práctica cultural" y, por el lado del programa de investigación $\mathrm{CdA}$, la formulación reciente de las diferentes nociones de "apropiación mutua" entre actores y la integración de esta noción de "práctica cultural".

Con respecto a esta primera serie de fenómenos, remitiré a un capítulo de libro (Theureau, 2000) que resumiré de manera drástica. Tangencialmente, esto me dará la oportunidad, como lo anuncié (en la última sub-sección de la sección 2), de dar, al pasar, un panorama muy parcial de lo que podrían ser una explicitación y una comparación de los análisis en términos de "modelo de flujo de información" y de "actividad-signo". Con respecto a esta segunda serie de fenómenos, remitiré a 
publicaciones de investigaciones empíricas de Edwin Hutchins y de sus colaboradores/ as y a uno de mis manuscritos (Theureau, 2019), referido a la crítica de la economía política desde el punto de vista de la actividad humana, donde tuve que desarrollar en términos de "apropiación mutua" las nociones de apropiación ya propuestas en el pasado.

La tercera serie de fenómenos, por su parte, enriquece los fenómenos considerados en el pasado por el programa de investigación CdA. Se trata aquí de superar los límites descriptivos y explicativos de la manera de considerar, en el programa de investigación $\mathrm{CdA}$, la única parte de las actividades humanas que da lugar a conciencia prerreflexiva (o experiencia), que fue objeto de críticas por parte de Edwin Hutchins desde el inicio. Esa superación comenzó a efectuarse recientemente en ciertas investigaciones deportivas en términos de "curso de in-formación" y de "articulación colectiva de los cursos de in-formación". Con respecto a esta tercera serie de fenómenos, que es objeto actualmente de numerosas y variadas investigaciones empíricas, remitiré a algunas publicaciones recientes.

El cúmulo de los análisis de estas tres series de fenómenos constituye un componente de un "programa de investigación aumentado" que podemos caracterizar como el inicio de un estudio sistemático de las relaciones entre las actividades individualescolectivas y colectivas-individuales que dan lugar y no dan lugar a experiencia para los actores.

39 A continuación abordaré en este orden estas tres series de fenómenos así acumulados y concluiré brevemente esta sección proponiendo una reflexión sobre la relación que mantuvieron las tres series de fenómenos puestas en evidencia (y más ampliamente, los avances logrados en el marco de estos dos programas de investigación) con las características de las situaciones estudiadas que los ocasionaron.

\subsection{El análisis de los fenómenos de des-colectivización}

Si bien la colectivización de las actividades humanas como articulación colectiva de las actividades humanas, debería ser analizada, su des-colectivización también debería serlo, si quisiéramos comprender lo que sucede en un colectivo de actores. Es por ello que, en Theureau (2000), sometí los mismos datos de actividad de conducción incidental-accidental de un reactor nuclear en simulador a escala plena de una versión provisoria de sala de comando, a un doble análisis, en términos de "actividad-signo" y en términos de "modelos de flujo de información", para resaltar el beneficio descriptivo y explicativo del primero relativamente al segundo. Esos datos eran insuficientes en muchos aspectos desde el punto de vista del programa de investigación CdA [grabaciónvideo del comportamiento de los tres actores involucrados y debriefing habitual al final de la simulación, estudio etnográfico previo limitado por parte de los/las investigadores/as, [ver Jeffroy, Theureau y Vermersch (1998) para los detalles], pero suficientes desde el punto de vista del programa de investigación 'cognición distribuida' por medio de un estudio etnográfico suficiente.

41 Mostré que, en el simulador a escala plena de la sala de control nuclear en situación incidental-accidental, las actividades de los tres actores principales [Operador-Reactor (OPR), Operador-Agua-Vapor (OAV) y Supervisor (SUP)], que eran supuestamente colectivas, se parecían más a las de los personajes del filme de Alain Resnais, "El año pasado en Maienbad": estos actores eran en parte de culturas diferentes, se cruzaban, 
pero rara vez se encontraban, aún cuando estaba más que previsto en los procedimientos y, por así decirlo, no miraban la película, no tenian tiempo o no se lo hacían para ponerse de acuerdo en caso de desacuerdo, por lo tanto, luego, no efectuaban las mismas selecciones de la información disponible durante sus acciones y construían saberes que estaban lejos de ser enteramente compartidos [23].

\subsection{El desarrollo de las nociones de apropiación mutua, la integración de la noción de "práctica cultural» y los fenómenos de construcción de un «sistema de cognición distribuida" (o "unidad de ecología cognitiva")}

42 La integración del paradigma de la enacción y el desarrollo de la noción sintética de "práctica cultural" en el programa de investigación 'cognición distribuida' y su asociación con las nociones de apropiación mutua introducidas recientemente en el programa de investigación CdA permiten dar cuenta de la génesis de un "sistema de cognición distribuida" (o "unidad de ecología cognitiva"). De esta forma; a) un "sistema de cognición distribuida" (o "unidad de ecología cognitiva") constituye a la vez una limitación y un efecto de procesos de apropiación individual y mutua, que resultan en sí de las actividades individuales-colectivas de los actores y dan lugar o no a conciencia prerreflexiva (o experiencia) para esos actores, por consiguiente, como "prácticas culturales" que superan los límites del objeto teórico 'curso de acción', y la descripción de su dinámica puede ser explicativa solamente dentro de los límites de la realización de esta apropiación individual y mutua; (b) cuando el resultado de esta apropiación individual y mutua es insuficiente para que la interacción entre los actores no dé lugar a malentendidos, comprobados o no por ellos, es necesario y posible analizar la emergencia de dichos malentendidos y los procesos de apropiación mutua eventualmente implementados por los actores para sobrellevarlos.

Con respecto a la definición de la "práctica cultural", mencionada (en sección 1) a partir de Hutchins (2008a), observé que una "práctica cultural" de esas características, no incluye necesariamente una comunicación simbólica y no da necesariamente lugar a una forma cualquiera de conciencia en los actores involucrados. Hay que agregar aquí, evidentemente, que, en consecuencia, dicha práctica cultural no da necesariamente lugar a conciencia prerreflexiva (o experiencia), aunque fuera solamente en parte, en esos mismos actores. Como ilustración de la noción de apropiación mutua propuse la de una pareja de bailarines de tango (Theureau, 2019) que dio lugar a un estudio de Serge Leblanc (comunicación personal). Esta ilustración vale también para la noción de "práctica cultural".

Sin tales nociones (práctica cultural, apropiación mutua), ni la actividad individualcolectiva como enacción y la dinámica del "sistema de cognición distribuida" (o "unidad de ecología cognitiva"), ni las descripciones en términos de "modelos de flujo de información" y aquellas en términos de "actividad-signo", no podrían ser relacionadas entre sí, sino solamente ser comparadas; y las divergencias comprobadas sólo podrían remitirse a la divergencia entre los paradigmas (cognición distribuida versus enacción) y a la divergencia entre los objetos teóricos ("sistema de cognición distribuida" (o "unidad de ecología cognitiva") versus "articulación colectiva de actividades individuales colectivas" que es la consecuencia de la primera. 


\subsection{Las investigaciones sobre los 'cursos de in-formación' y su articulación colectiva como superación del programa de investigación $\mathrm{CdA}$ a la vez que del programa de investigación 'cognición distribuida'}

Hemos visto por un lado (en Introducción) que el programa de investigación 'cognición distribuida' había encontrado la hipótesis de la enacción a partir de investigaciones sobre la cognición encarnada llevadas a cabo a partir de 2005. Hemos visto por otro lado (en la sección 2) que, durante el mismo período, comenzaron a desarrollarse en el programa de investigación $\mathrm{CdA}$ investigaciones deportivas sobre los cursos de información y la articulación colectiva de los 'cursos de in-formación' dentro de los límites de los datos disponibles sobre los comportamientos, los parámetros fisiológicos y los procesos materiales (inflexión independiente 4). Un nuevo punto de encuentro privilegiado apareció entonces entre los dos programas de investigación: el que se ubica entre las investigaciones sobre la cognición encarnada (del lado del programa de investigación 'cognición distribuida') y las investigaciones sobre los 'cursos de información' y su articulación colectiva, en las cuales la descripción de las actividades y de su articulación colectiva va más allá de la de las actividades que dan lugar a conciencia prereflexiva (del lado del programa de investigación 'curso de acción').

La diferencia entre los 'cursos de experiencia' (por consiguiente también las actividades que dan lugar a conciencia prerreflexiva o experiencia en cada instante) de los actores y el conjunto de las actividades, ya sea que dé lugar o no a conciencia prerreflexiva (o experiencia), de esos mismos actores, que separaba los dos programas de investigación, se vuelve a encontrar ahora dentro del mismo programa de investigación CdA, en este caso, en su parte de estudio de los 'cursos de in-formación' y de su articulación colectiva. Las investigaciones deportivas sobre los 'cursos de in-formación' y su articulación colectiva consideran en efecto al mismo tiempo, la conciencia prerreflexiva (o experiencia) de los actores y detalles de su cognición encarnada y procesos materiales en el entorno que son ajenos a esta conciencia prerreflexiva (o experiencia). Al respecto, remitiré, por ejemplo, a las publicaciones siguientes: GalPetitfaux, Adé, Poizat y Seifert (2013); R'Kiouak (2017); R'Kiouak, Saury y Bourbousson (2018); Seifert, Lardy, Bourbousson, Adé, Nordez, Thouvareq, et al. (2017); Terrien, Huet, Iachkine y Saury (2020).

\subsection{Las situaciones privilegiadas para tal o cual desarrollo científico}

Volvamos, para cerrar esta sección, a la forma con la que mostré en párrafos anteriores la necesidad de superar el límite del programa de investigación 'cognición distribuida' que era la de ignorar los fenómenos de des-colectivización de las actividades humanas: apoyándome en una investigación sobre las actividades de los operadores en un simulador a escala plena de una sala de control de reactor nuclear. Los fenómenos de des-colectivización y los diversos fenómenos de la actividad de los operadores descritos por la concatenación de signos hexádicos, señalados en esta ocasión, escapan efectivamente al objeto teórico 'cognición distribuida' y a su descripción en términos de "flujo de información". Pero, si consideramos las publicaciones de las investigaciones llevadas a cabo por Edwin Hutchins y sus colaboradores/as sobre la cognición 
distribuida en la cabina (cockpit) (Hutchins, 1994, 2000; Hutchins \& Klausen, 1998) [24], poniendo entre paréntesis las hipótesis, nociones analíticas y datos empíricos del programa de investigación $\mathrm{CdA}$, no podemos encontrar sino convincentes las descripciones de la cognición distribuida en términos de "flujo de información" que se efectuaron y sirven a la vez de ilustración y de puesta a prueba empírica del programa de investigación 'cognición distribuida'. Hasta podemos decir que las descripciones de la cognición distribuida en términos de "modelo de flujo de información" realizadas por el programa de investigación 'cognición distribuida' aparecen entonces, desde el punto de vista del programa de investigación $\mathrm{CdA}$, como reducciones operatorias locales limitadas, que son útiles para resaltar los fenómenos de cognición distribuida y/ o suficientes para contribuir a eventuales proyectos de ingeniería de las situaciones consideradas.

Me parece que esta constatación ilustra de nuevo el interés de la noción de "situación privilegiada para la resolución de tal o cual problema científico", que fue propuesta desde el inicio en el marco de la epistemología del programa de investigación CdA a fin de desempeñar el rol que las situaciones experimentales desempeñan en el desarrollo de los programas de investigación experimentales. De dicha constatación, podemos inferir, sin equivocarnos, que los fenómenos de des-colectivización aparecen con mayor facilidad ya sea en una sala de control de reactor nuclear, o en una sala de control del tráfico ferroviario, o en una unidad de cuidados de un hospital, es decir en situaciones estudiadas en el marco del programa de investigación $\mathrm{CdA}\left[{ }^{25}\right]$ en sus inicios, que en una cabina donde los actores están confinados en un espacio reducido.

Si consideramos sucesivamente las otras situaciones que se abordaron en esta sección, podemos decir igualmente que: la situación de nadadores que se entrenan con un dispositivo de evaluación de su rendimiento (Gal-Petitfaux, Adé, Poizat, \& Seifert, 2013) es una situación privilegiada para estudiar los cursos de in-formación individuales; la situación de una pareja de bailarines de tango en una pista de baile, lo es para estudiar las prácticas culturales y la apropiación mutua; las situaciones de remo en un equipo de dos remeros (R'Kiouak, 2017; R'Kiouak, Saury, \& Bourbousson, 2018; Seifert, Lardy, Bourbousson, Adé, Nordez, Thouvareq, et al., 2017) y las situaciones de conducción colectiva de veleros "volantes" (Terrien, Huet, Iachkine, \& Saury, 2020), lo son para estudiar la articulación colectiva de los cursos de in-formación.

De allí el interés de acumular las situaciones privilegiadas por los dos programas de investigación en el pasado y de generalizar esta consideración de las "situaciones privilegiadas para la resolución de tal o cual problema científico" en el futuro.

\section{La ingeniería de las situaciones y la ingeniería de las herramientas cognitivas}

51 Un sinnúmero de investigaciones llevadas a cabo en el marco de los programas de investigación 'cognición distribuida' y 'curso de acción' tuvieron un componente tecnológico y dieron lugar a contratos con empresas. Es un punto común de ambos programas de investigación que está lejos de ser compartido por el conjunto de las investigaciones en ciencias humanas y sociales, incluidas aquellas que son psicológicas y cognitivas. Por el lado de 'cognición distribuida', citemos primero las publicaciones sobre el componente tecnológico de las investigaciones sobre la navegación de los buques de guerra, que tienen la particularidad de estar referidas a la capacitación en 
simulador (Halff, Hollan, \& Hutchins, 1986; Hollan \& Hutchins, 1984). Sucede lo mismo con las investigaciones posteriores sobre la cabina (cockpit), el control aéreo, la conducción de un automóvil, etc. En Hollan y Hutchin (2009), Hollan, Hutchins y Kirsh (2000) y, Hutchins y Hollan (1986), los autores presentan todo un abanico de herramientas cognitivas que constituye un estado de lo que podemos calificar de "ergonomía cognitiva en relación orgánica con el programa de investigación 'cognición distribuida".

Si Edwin Hutchins y sus colaboradores/as conciben estas herramientas cognitivas como si debieran insertarse en "sistemas de cognición distribuida", las investigaciones CdA las conciben como si debieran insertarse en esos "sistemas de cognición distribuida" concebidos en términos de "situaciones colectivas (o más bien colectivasindividuales)", pero también en situaciones individuales-colectivas, entre las cuales figuran las de formación y educación. Las investigaciones CdA insistieron en la concepción global de las situaciones, ya sea individuales-colectivas o colectivasindividuales, y no presentaron, al contrario de las de Jim Hollan y Edwin Hutchins, herramientas cognitivas concebidas como genéricas. Además, también permitieron la concepción de herramientas cognitivas que presumiblemente valen en diversas situaciones o familias de situaciones (por ejemplo, últimamente, en el caso de las ayudas para la escucha activa y situaciones de escucha activa: Goldszmidt, Donin, \& Theureau, 2007; Goldszmitdt, \& Theureau, 2010).

Podemos, de conformidad con el paradigma de la enacción, reformular a la vez el lado tecnológico del programa de investigación 'cognición distribuida' y reconducir el lado tecnológico de la parte colectiva del programa de investigación CdA en términos de "programa de investigación tecnológica en ingeniería de las situaciones colectivas, es decir colectivas-individuales, como incluyendo a los actores y su entorno intersticial". Evidentemente, podemos también precisar el "programa de investigación tecnológica en ingeniería de las situaciones individuales-colectivas" insistiendo en el hecho de que cada actor considerado se sitúa en un entorno compuesto poor otros actores, por el entorno intersticial entre ese actor y aquellos otros actores y por el entorno más amplio que engloba a todos esos actores. Hasta ahí, fuera de la ingeniería de las situaciones de formación, de educación y de entrenamiento, la vertiente de investigación tecnológica del programa de investigación $\mathrm{CdA}$ sólo fue objeto de una obra de síntesis, ya antigua, centrada en la ergonomía (Theureau \& Jeffroy, 1994, retomada en parte en Theureau, 2015b). Los desarrollos que se realizaron desde entonces, y que sobrepasan ampliamente la ergonomía, y aquellos de los que acabo de hablar, y que están vigentes o a la espera de realización, deberían constituir la materia de una nueva síntesis.

Si el programa de investigación 'cognición distribuida' tomó en cuenta los "saberes de la cabina", lo hizo muy modestamente, y si se conocieron consecuencias en materia de ingeniería de las situaciones de formación, educación y entrenamiento, estas no fueron publicadas. Lo esencial de la ingeniería de las situaciones de formación, educación y entrenamiento desarrollada en el programa de investigación CdA, de la que Marc Durand señaló las especificidades respecto de la ingeniería de las situaciones en general (Durand, 2008), debe ser pues repensado. Varios libros sobre el estado de la cuestión (Yvon, \& Durand, 2012; Bourgeois, \& Durand, 2012; Poizat, Durand, \& Theureau, 2016; Yvon \& Durand, 2012) y de síntesis (Saury, Adé, et al., 2013), sobre el tema ya fueron publicados. Sin embargo, sería oportuno enriquecer el tema en términos de "saberes de 
la cabina" o del "sistema de cognición distribuida" (o "unidad de ecología cognitiva") y por lo tanto de ingeniería de la formación, educación y entrenamiento de la "cabina" o del "sistema de cognición distribuida" (o "unidad de ecología cognitiva").

\section{El análisis de las actividades de investigación científica y tecnológica, de las actividades de creación artística y, más generalmente de las actividades creativas.}

55 El programa de investigación 'cognición distribuida' abordó las actividades de investigación científica en períodos muy cortos. El programa de investigación CdA abordó por su parte, las actividades artísticas y lo hizo, en relación con las actividades de composición musical, en períodos más largos. Como las actividades de investigación científica y las de composición musical se diferencian en muchos aspectos, podemos apoyarnos en el estudio de unas para abordar el de las otras. Los logros de ambos programas de investigación pueden entonces completarse con el abordaje de las actividades de creación en toda su generalidad (ver Theureau, 2017) [ $\left.{ }^{26}\right]$.

\subsection{Las actividades de investigación científica de lo instantáneo al largo plazo, sin olvidar el mediano plazo.}

Según Alac y Hutchins (2004) "la conducción de las investigaciones científicas incluye numerosos tipos de procesos cognitivos: procesos internos [a los actores] como dos que hicieron foco en la ciencia cognitiva durante décadas (...); procesos que se dan cuando se propagan representaciones a través de los medios representacionales; otros son procesos ampliamente distribuidos que intervienen en la circulación de las adhesiones y en la difusión de las ideas a través de las comunidades científicas (...) [citación, entre otros, de Fleck, [1935], 1979, 2005)]. Consideraremos un tipo diferente de procesos cognitivos que (...) se producen en las interacciones entre los investigadores científicos y con las representaciones materiales. No son procesos internos que acompañan el comportamiento observado; mostraremos más bien que resulta útil considerar las interacciones como procesos cognitivos" (op.cit., p. 629-630, traducción libre) [27].

Las diferentes publicaciones en términos de 'cognición distribuida' relativas a actividades de investigación (Alac, \& Hutchins, 2004; Becvar, Hollan, \& Hutchins, 2008; Hutchins, 2012) dentro de los límites así determinados, no consideran, sin embargo, estas interacciones sino a muy corto plazo. Los procesos ejemplificados por Fleck ([1935], 1979, 2005), así como por Holton (1973), sólo pueden ser estudiados en el marco de un desarrollo del programa de investigación $\mathrm{CdA}$ en términos de análisis multiniveles de la actividad humana en relaciones de par* (ver sección 2) o en su equivalente. Pero el estudio de la articulación colectiva de las actividades individualescolectivas de un conjunto de actores científicos (investigadores y otros) dotados de su entorno intersticial puede a priori superar ese muy corto plazo interesándose en un mediano plazo, de algunos días, algunos meses o algunos años, si se extiende al conjunto de las actividades de investigación y, más generalmente aún, a todas las actividades de creación, lo que ya se hizo en diversas investigaciones sobre las actividades de composición musical. 


\subsection{De las actividades de composición musical a las actividades creativas en general}

58 Aunque el programa de investigación CdA no abordó este análisis empírico de las actividades de investigación científica, los análisis desarrollados con relación a las actividades de composición musical dan paso a su estudio en los medianos plazos mencionados. Estos análisis trataron sobre la actividad de composición musical durante cinco años de dos obras asociadas de Philippe Leroux, Voi(rex) y Apocalypsis (Donin, \& Theureau, 2007, 2008, 2015; Theureau, \& Donin, 2006), así como sobre la actividad conjunta durante dos años de composición de una obra para cuarteto por parte de Florence Baschet, Streicherkreis, y de diseño de un dispositivo informático por parte de un equipo de investigación sobre el gesto musical (Donin, 2017; Donin, Goldszmidt \& Theureau, 2009). Estos análisis, prolongados por los de otras actividades de composición musical (Donin, \& Ferron, 2012) y los de otros autores, dieron lugar a una síntesis (Donin, 2018), que insiste sobre la complementariedad entre estos análisis a medianos plazos y análisis históricos a largo plazo. Estos últimos pueden ser ejemplificados por Fleck $([1935], 1979,2005)$ y Holton $(1973,1981)$, ya citados precedentemente en lo relativo a las actividades de investigación científica.

\section{El programa de investigación 'curso de acción' aumentado, su articulación con las investigaciones sobre la actividad animal como enacción y dinámica cultural y la continuación del debate con las neurociencias.}

Se trata, por último, haciendo uso del beneficio de la integración del paradigma de la enacción por el programa de investigación 'cognición distribuida', (1) de aprovechar la extensión, por medio del programa de investigación 'cognición distribuida' de las investigaciones en las actividades animales; (2) de continuar el debate con las neurociencias que empezó Edwin Hutchins. Abordemos sucesivamente estos dos puntos en esta última sección.

\subsection{El análisis de las actividades animales y la cuestión de la emergencia del lenguaje}

El observatorio de la cognición distribuida, al no recurrir de manera controlada a verbalizaciones de los actores como lo hace el de los cursos de acción, daba lugar a considerar tanto la actividad animal como la actividad humana. La integración del paradigma de la enacción, supuestamente referida a todo ser vivo, de la garrapata al hombre, sólo podía reforzar esta apertura en la Etapa 3 de desarrollo de este programa de investigación (ver sección 1). Las investigaciones sobre la cognición animal realizadas durante la Etapa 2 muestran en efecto, retrospectivamente, el interés y al mismo tiempo la insuficiencia de considerar el colectivo y el entorno si no se considera también el carácter encarnado de la cognición. Pienso en la tesis de doctorado (PhD) de 
Deborah Forster bajo la dirección de Edwin Hutchins sobre la cognición de hordas de mandriles (ver, por ejemplo, Sturm, Forster, \& Hutchins, 1997).

El hecho de considerar la cognición encarnada y la integración del paradigma de la enacción permitieron efectivamente renovar esas investigaciones en la Etapa 3 (Hutchins, 2008a; Hutchins, \& Johnson, 2009). Es la noción de "práctica cultural” (por su definición, ver también sección 1) lo que se ubica en el centro de dichas investigaciones. Si bien es cierto que existen prácticas culturales simbólicas, por lo menos en el hombre, esta noción de "práctica cultural" es, como lo hemos visto, más amplia. En Hutchins (2008), la noción de "práctica cultural" permite la crítica de interpretaciones en términos de acceso a lo simbólico de chimpancés en cautiverio que no recibieron capacitación de lenguaje -al principio, Edwin Hutchins (comunicación personal) era entusiasta hasta que se dio cuenta de que la noción de práctica cultural construida entre esos chimpancés y sus guardias y experimentadores bastaba para interpretar los fenómenos observados y que no era entonces necesario postular el acceso de estos chimpancés a lo simbólico-, mientras que, en Hutchins y Johnson (2009), esa noción permite mostrar en chimpancés la "complementariedad de la acción sumada a la imitación, de lo icónico sumado al gesto indexical, de la coordinación entre múltiples modalidades sensoriales y perceptivas, y de la orquestación de las coordinaciones motrices intra- e inter-individuales" (Hutchins \& Johnson, 2009, p. 523, traducción libre). Podemos observar que este segundo texto milita a favor de una fenomenología de la actividad animal que sea también una semiótica, en este caso una semiótica de la actividad, como lo es la teoría de la actividad-signo (en la cual la parte no-simbólica puede extenderse del hombre al animal).

Como en el caso de las actividades de investigación, es necesario para estas actividades animales ampliar el horizonte temporal de las investigaciones. Habría que interesarse también en las relaciones entre las prácticas culturales, la cultura y los diferentes tipos de apropiación mutua (ver sección 4). Estas investigaciones sobre la articulación colectiva de las actividades animales y/o animales y humanas se beneficiarían si fueran desarrolladas en relación orgánica con una ingeniería de las situaciones animales y/o compartidas entre hombres y animales. Por último, aún en sus límites actuales, las investigaciones llevadas a cabo en el marco del programa de investigación 'cognición distribuida' sobre la cognición animal, muestran el interés teórico y heurístico de una relación entre tales investigaciones y aquellas sobre la cognición humana, que se encarna, se sitúa y se cultiva como la cognición animal, pero de manera en parte diferente.

Pero, como por una parte, un "programa de investigación 'curso de acción' aumentado" sólo puede incluir en su núcleo teórico la hipótesis de la conciencia prerreflexiva (o experiencia) y en su núcleo metodológico los métodos de acceso a esa conciencia prerreflexiva (o experiencia), y como, por otra parte, una extensión de la teoría de la actividad-signo a la actividad animal exigiría que sea precisada una noción de conciencia en el animal, susceptible de ser documentada empíricamente, lo que plantea numerosos problemas no resueltos [28], hoy en día podemos considerar que no se trata tanto de incluir en este "programa de investigación 'curso de acción' aumentado" el estudio de las actividades animales, sino de desarrollar sus relaciones, y hasta sus articulaciones en las situaciones privilegiadas que son, desde este punto de vista, las situaciones de interacción hombre-animal, con investigaciones sobre la actividad animal realizadas en el marco de otros programas de investigación, y muy 
particularmente, el programa de investigación 'cognición distribuida' revisado después de integrar la hipótesis de la enacción, es decir la 'nueva cognición distribuida' prometida por Edwin Hutchins (ver Introducción).

\subsection{Las actividades humanas y animales y el debate con las neurociencias}

En el marco de la lucha universitaria por acaparar los financiamientos, la creatividad actual y las expectativas de las neurociencias generaron pretensiones por monopolizar las investigaciones sobre la cognición (a la vez humana y animal, puesto que humanos y animales son aquí objeto de experimentaciones de laboratorio) por parte de los investigadores en neurociencias. Hutchins (2011) habla, por el contrario, de "cognición culta" y recuerda que "el cerebro posee poderes causales, pero en materia de cognición humana, la mayoría de los poderes causales del cerebro humano derivan de la experiencia adquirida anteriormente en prácticas culturales" [ $\left.{ }^{29}\right]$. Asimismo, este olvido de las prácticas culturales es lo que Edwin Hutchins reprocha a la corriente llamada de la "extended mind" (Clark, \& Chalmers, 2010).

Una vez reparado este olvido, la antropokogia y las neurociencias oueden mantener un debate fructuoso. Este debate inaugurado por Edwin Hutchins con las neurociencias me parece vital en Francia, muy particularmente en ciencias de la formación, de la educación y del entrenamiento, donde el monopolio de las neurociencias, y hasta el cuasi-monopolio dentro de dichas neurociencias de los programas de investigación en términos de modelos mentales predefinidos de un entorno predefinido, que se oponen directamente a la hipótesis de la enacción, inspiran de buen grado los discursos del actual Ministerio de Educación Nacional. Asimismo, por otra parte, la experimentación psicofisiológica de laboratorio inspira de manera dominante por no decir monopólica la economía política neoclásica contemporánea, ella misma ampliamente dominante si no monopólica, y en consecuencia también, la enseñanza secundaria y superior de la economía política en Francia (ver Theureau, 2019, ya citado). Me parece que la continuación de este debate con las neurociencias a partir de los avances de las investigaciones sobre las actividades humanas debería formar parte de este "programa de investigación 'curso de acción' aumentado". Concluiré sobre este punto.

\section{BIBLIOGRAFÍA}

Alac, M., \& Hutchins, E. (2004). I see what you are saying: action as cognition in fMRI brain mapping practice. Journal of Cognition \& Culture, 4(3-4), 629-661. https://doi.org/ $10.1163 / 1568537042484977$

Ball, L., \& Ormerod, T. (2000). Putting ethnography to work: the case for a cognitive ethnography of design. International Journal of Human-Computer Studies, 53(1), 147-168. https://doi.org/10.1006/ ijhc. 2000.0372 
Becvar, A., Hollan, H., \& Hutchins, E. (2008). Representational gestures as cognitive artifacts for developing theories in a scientific laboratory. In M. Ackerman, C. Halverson, T. Erickson, \& W. Kellogg (Eds.), Resources, co-evolution and artifacts: theory in CSCW (pp. 117-143). London : Springer.

Bourbousson, J., \& Sève, C. (2010). Analyse de la performance collective, nouveau terrain d'expression de la théorie des systèmes dynamiques. STAPS, 90(4), 59-74. https://doi.org/10.3917/ sta.090.0059

Bourbousson, J., Sève, C., \& McGarry, T. (2010a). Space-time coordination dynamics in basketball : Part 1. Intra- and inter-couplings among player dyads. Journal of Sports Sciences, 28(3), 339-347. https://doi.org/10.1080/02640410903503632

Bourbousson, J., Sève, C., \& McGarry, T. (2010b). Space-time coordination dynamics in basketball : Part 2. The interaction between the two teams. Journal of Sports Sciences, 28(3), 349-358. https://doi.org/10.1080/02640410903503640

Bourgeois, E., \& Durand, M. (Eds.)(2012). Apprendre au travail. Paris : PUF.

Button, G. (2008). Against “distributed cognition”. Theory, Culture \& Society, 25(2), 87-104. https:// doi.org/10.1177/0263276407086792

Clarke, E., Doffman, M., \& Lim, L. (2013). Distributed creativity and ecological dynamics: a case study of Liza Lim's “Tongue of the invisible”. Music and Letters, 94(4), 628-663. https://doi.org/ $10.1093 / \mathrm{ml} /$ gct118

Clark, A., \& Chalmers, D. (2010). The extended mind. In R. Menary (Ed.), The extended mind (pp. 27-42). Cambridge : MIT Press.

Dieumegard, G., \& Nogry, S. (2018). Experienced relations and structured inscriptions. EARLI SIG 17 Méthodes in learning \& SIG 25 Educational theories, August 26th-28th, Cambridge, United Kingdom.

Dillenbourg, P., Baker A., Blaye, A., \& O’Malley, C. (1996). The evolution of research on collaborative learning. In E. Spade, \& P. Reiman (Eds.), Learning in humans and machines: Towards an interdisciplinary learning science (pp. 189-211). Oxford : Elsevier.

Donin, N. (2017) Domesticating gesture: the collaborative creative process of Florence Baschet's Streicherkreis for 'augmented' string quartet (2006-2008). In E. Clarke, \& M. Doffman (Eds.), Distributed creativity: collaboration and improvisation in contemporary music (pp. 70-87). New York : Oxford University Press.

Donin, N. (2018). La musicologie des processus de composition : Entre histoire et cognition. Transposition, H-S1. https://doi.org/10.4000/transposition.1689

Donin, N., \& Feron, F.-X. (2012). Tracking the composer's cognition in the course of a creative process: Stefano Gervasoni and the beginning of Gramigna. Musicae Scientiae, 16(3), 262-285. https://doi.org/10.1177/1029864912448328

Donin, N., Goldszmidt, S., \& Theureau, J. (2009). Organiser l'invention technologique et artistique ? L'activité collective de conception conjointe d'une œuvre et d'un dispositif informatique pour quatuor à cordes. Activités, 6(2), 24-43. https://doi.org/10.4000/activites.2253

Donin, N., \& Theureau, J. (2007). Theoretical and methodological issues related to long term creative cognition: the case of musical composition. Cognition, Technology \& Work, 9(4), 233-251. https://doi.org/10.1007/s10111-007-0082-z

Donin, N., \& Theureau, J. (2008). L'activité de composition musicale comme exploitation/ construction de situations : Anthropologie cognitive de la composition d'une œuvre musicale par 
Philippe Leroux. Intellectica, 48-49, 175-205. https://intellectica.org/fr/l-activite-de-compositioncomme-exploitationconstruction-de-situations

Donin, N., \& Theureau, J. (2008). La coproduction des œuvres et de l'atelier par le compositeur (à partir d'une étude de l'activité créatrice de Philippe Leroux entre 2001 et 2006). Circuit : Musiques contemporaines, 18(1), 59-71. https://doi.org/10.7202/017909ar

Donin, N., \& Theureau, J. (2015). Le sentiment de la forme : analyse génétique et cognitive de la composition d'un mouvement d'Apocalypsis par Philippe Leroux. In N. Donin, A. Grésillon, \& J.-L. Lebrave (Eds.), Genèses musicales (pp. 101-128). Paris : Presses Universitaires de Paris-Sorbonne.

Dubbels, B. (2011). Cognitive ethnography: A methodology for measure and analysis of learning for game studies. International Journal of Gaming and Computer-Mediated Simulations, 3(1), 68-78. https://doi.org/10.4018/jgcms.2011010105

Dufresne, R. (2001). Le contrôle des environnements dynamiques : étude ergonomique dans une perspective d'automatisation d'un système de contrôle de la circulation ferroviaire. Thèse de doctorat, École Pratique des Hautes Études, Paris, France.

Durand, M. (2008). Un programme de recherche technologique en formation des adultes : Une approche enactive de l'activité humaine et de l'accompagnement de son apprentissage/ développement. Éducation et Didactique, 2(3), 97-121. https://doi.org/10.4000/ educationdidactique.373

Fauconnier, G., \& Turner, M. (2002). The way we think - Conceptual blending and the mind's hidden complexity. New York : Basic books.

Fleck, L. ([1935], 1979) Genesis and development of a scientific fact. Chicago : Univ. of Chicago Press. [Traduction française : (2005) Genèse et développement d'un fait scientifique. Paris : Les Belles Lettres] Fouse, A., Hollan J. (2010). Dataprism: a tool for visualizing multimodal data. MB'10: Proceedings of the 7th International Conference on Methods and Techniques in Behavioral Research (pp. 1-4). https:// doi.org/10.1145/1931344.1931345

Fouse A., Weibel, N., Hutchins, E., \& Hollan, J. (2011). ChronoViz: a system for supporting navigation of time-coded data. CHI '11 Extended Abstracts on Human Factors in Computing Systems (pp. 299-304). https://doi.org/10.1145/1979742.1979706

Gal-Petitfaux, N., Adé, D., Poizat, G., \& Seifert, L. (2013). L'intégration de données biomécaniques et d'expérience pour comprendre l'activité et concevoir un dispositif technologique : étude d'une situation d'évaluation avec des nageurs de haut niveau. Le Travail Humain, 76(3), 257-282. https:// doi.org/10.3917/th.763.0257

Goldszmidt, S., Donin, N., \& Theureau, J. (2007). Navigation génétique dans une œuvre musicale. IHM'07 : Actes de la 19ème Conférence Interaction Homme-Machine (pp. 159-166). https:// doi.org/10.1145/1541436.1541467

Goldszmidt, S., \& Theureau, J. (2010). Conception de situations d'assistance à l'écoute musicale et analyse de l'activité de composition musicale. In G. Valléry, M.-C. Le Port, \& M. Zouinar (Eds.), Ergonomie, conception de produits et services médiatisés (pp. 157-182). Paris : PUF. Gorman, J., Dunbar, T., Grimm, D., \& Gipson, C. (2017). Understanding and modeling teams as dynamical systems. Frontiers in Psychology, 8, 1053 https://doi.org/ 10.3389/fpsyg.2017.01053 Grison, B. (1998). Structures de raisonnement dans un laboratoire de neurobiologie du développement : étude dans une perspective d'écologie cognitive. Thèse de doctorat, EHESS, Paris, France.

Halbwachs, M. (1925). Les cadres sociaux de la mémoire. Paris : Librairie Alcan. 
Halff, H., Hollan, J., \& Hutchins, E. (1986). Cognitive science and military training. American Psychologist, 41(10), 1131-1139. https://doi.org/10.1037/0003-066X.41.10.1131

Haradji, Y., Guibourdenche, J., Reynaud, Q., Poizat, G., Sabouret N., Sempé, F., Huraux, Th., \& Galbat, M. (2018). De la modélisation de l'activité humaine à la modélisation pour la simulation sociale : entre réalisme et fécondité technologique. Activités, 15(1). https://doi.org/10.4000/ activites.3106

Haué, J.-B. (2005) Comparing distributed cognition and course of action : an application to car driving. EACE'2005: Proceedings of the Annual Conference of the European Association of Cognitive Ergonomics (pp. 139-146). https://doi.org/10.5555/1124666.1124685

Haviland, J. (2011). Musical spaces. In J. Streeck, C. Goodwin, \& C. Le Baron (Eds.), Embodied interaction, language and body in the material world (pp. 289-304). New York: Cambridge University Press.

Hollan, J., Hutchins, E., \& Weitzman, L. (1984). STEAMER: an interactive inspectable simulationbased training system. AI Magazine, 5(2), 15-27. https://doi.org/10.1609/aimag.v5i2.434

Hollan, J., \& Hutchins, E. (2009). Opportunities and challenges for augmented environments: a distributed cognition perspective. In S. Lahlou (Ed.), Designing user friendly augmented work environments: From Meeting Rooms to Digital Collaborative Spaces (pp. 237-259). London : Springer.

Hollan, J., Hutchins, E., \& Kirsch, D. (2000). Distributed cognition: toward a new foundation for human-computer interaction research. ACM Transactions on Computer-Human Interaction, 7(2), 174-196. https://doi.org/10.1145/353485.353487

Holton, G. (1973). Thematic origins of scientific thought - Kepler to Einstein. Cambridge : Harvard University Press.

Holton, G. (1981). L'imagination scientifique. Paris : Gallimard.

Hutchins, E. (1980). Culture and inference: a Trobriand case study. Cambridge : Harvard University Press.

Hutchins, E. (1983). Understanding micronesian navigation. In D. Gentner, \& A. Stevens (Eds.), Mental models (pp. 191-225). Hillsdale: Lawrence Erlbaum.

Hutchins, E. (1994). Comment le cockpit se souvient de ses vitesses. Sociologie du Travail, 46(4), 451-473.

Hutchins, E. (1995). Cognition in the wild. Cambridge : MIT Press.

Hutchins, E. (2000). Les conséquences cognitives des configurations du flux d'information. Intellectica, 30, 53-74.

Hutchins, E. (2005). Material anchors for conceptual blends. Journal of Pragmatics, 37(10), 1555-1577. https://doi.org/10.1016/j.pragma.2004.06.008

Hutchins, E. (2006). Imagining the cognitive life of things. In L. Malafouris, \& C. Renfrew (Eds.), The cognitive life of things: recasting the boundaries of the mind (pp. 91-101). Cambridge : McDonald Institute for Archaeological Research.

Hutchins, E. (2008a). The role of cultural practices in the emergence of modern human intelligence. Philosophical Transactions of the Royal Society B, 363(1499), 2011-2019. https://doi.org/ 10.1098/rstb.2008.0003

Hutchins, E. (2008b). Dew (Digital Ethnographer Workbench) Manifesto, Distributed Cognition and Human-Computer Interaction Laboratory (Dcog-HCI Lab), Department of Cognitive Science, 
University of California, San Diego (UCSD). Retrieved February 22, 2020, from http://

hci.ucsd.edu/research/dew/manifesto/

Hutchins, E. (2010a). Cognitive ecology. Topics in Cognitive Science, 2(4), 705-715. https://doi.org/

10.1111/j.1756-8765.2010.01089.x

Hutchins, E. (2010b). Enaction, imagination, and insight. In J. Stewart, O. Gapenne, \& E. Di Paolo (Eds.), Enaction: towards a new paradigm in cognitive science (pp. 425-450). Cambridge : MIT Press.

Hutchins, E. (2011). Enculturating the supersized mind. Philosophical Studies, 152(3),

437-446. https://doi.org/10.1007/s11098-010-9599-8

Hutchins, E. (2012). Concepts in practice as sources of order. Mind, Culture and Activity, 19(3), 314-323. https://doi.org/10.1080/10749039.2012.694006

Hutchins, E, \& Hazlehurst B. (1991). Learning in the cultural process. In C. Langton, C. Taylor, D. Farmer, \& S. Rasmussen (Eds.), Artificial life I I: Santa Fe Institute studies in the sciences of complexity (pp. 689-706). Redwood City: Addison Wesley.

Hutchins, E., \& Hazlehurst, B. (1995). How to invent a lexicon: the development of shared symbols in interaction. In N. Gilbert, \& R. Conte (Eds.), Artificial societies: the computer simulation of social life (pp. 157-189). London : UCL Press.

Hutchins, E., \& Holland, J. (1986). Direct manipulation interfaces. In D. Norman, \& S. Draper (Eds.) User centered design (pp. 87-124). London : Lawrence Erlbaum.

Hutchins, E., \& Johnson, C. (2009). Modeling the emergence of language as an embodied collective cognitive activity. Topics in Cognitive Science, 1(3), 523-546. https://doi.org/10.1111/j.

1756-8765.2009.01033.x

Hutchins, E., \& Klausen, T. (1998). Distributed cognition in an airline cockpit. In Y. Engeström, \& D. Middleton (Eds.), Communication \& cognition at work (pp. 15-34). Cambridge : Cambridge University Press.

Hutchins, E., Weibel, E., Emmenegger, C., Fouse, A., \& Holder, B. (2013). An integrative approach to understanding flight crew activity. Journal of Cognitive Engineering and Decision making, 7(4), 353-376. https://doi.org/10.1177/1555343413495547

Hutto, D., Kirchhoff, M., \& Myin, E. (2014). Extensive enactivism: why keep it all in? Frontiers in Human Neuroscience, 8, 706. https://doi.org/10.3389/fnhum.2014.00706

Jeffroy, F., Theureau, J., \& Vermersch, P. (1998). Quel guidage des opérateurs en situation incidentelleaccidentelle? Analyse ergonomique de l'activité de conduite avec procédures. Rapport DES SEFH $\mathrm{n}^{\circ} 2$, Fontenay aux Roses : IRSN.

Johnson, C. (2015). The cognitive ecology of dolphin social engagement. In D. Herzing, \& C. Johnson (Eds.), Dolphin Communication \& Cognition (pp. 229-256). Cambridge : MIT Press.

Kaastra, L. (2011). Annotation and the coordination of cognitive processes in western art music performance. In A. Williamon, D. Edwards, \& L. Bartel (Eds.), Proceedings of the International Symposium on Performance Science (pp. 675-680). Utrecht : European Association of Conservatoires (AEC).

Karnowski, J., Hutchins, E., \& Johnson, C. (2015) Dolphin detection and tracking. Proceedings of 2015 IEEE Winter Applications and Computer Vision Workshops (pp. 51-56). https://doi.org/10.1109/ WACVW.2015.10

Kuhn, T. (1962/1983). La structure des révolutions scientifiques. Paris : Flammarion 
Latour, B. (1995). "Cogito ergo sumus!” A review of Ed Hutchins Cognition in the wild, Mind, Culture and Activity, 3(1), 54-63. https://doi.org/10.1207/s15327884mca0301_

Lakatos I. (1970) Falsifications and the Methodology of Scientific Research Programmes. In I. Lakatos, A. Musgrave (Eds.), Criticism and the Growth of Knowledge (pp. 91-196). Cambridge: Univ. Press.

Malinowski, B. (1963). Les argonautes du pacifique. Paris: Gallimard.

Malinowski, B. (1985). Journal d'ethnographe. Paris : Le Seuil.

Perrin, N., Theureau, J., Menu, J., \& Durand, M. (2011). SIDE-CAR : Un outil numérique d'aide à l'analyse de l'activité par rétrodiction. Exploitation selon le cadre théorique du « cours d'action ». Recherches qualitatives, 30(2), 148-174. http://www.recherche-qualitative.qc.ca/ documents/files/revue/edition_reguliere/numero30(2)/RQ_30(2)_Perrin-al.pdf

Perrin, N., \& Vanini De Carlo, K. (2016). L'événementialisation comme co-construction des connaissances : esquisse d'une multi-méthode issue de l'analyse de l'activité et de l'analyse narrative. In F. Ligozat, M. Charmillot, \& A. Muller (Eds.), Le partage des savoirs dans les processus de recherche en éducation (pp. 251-271). Louvain-la-Neuve : De Boeck.

Pinsky, L. (1992). Concevoir pour l'action et la communication : essais d'ergonomie cognitive. Berne : Peter Lang.

Pinsky, L., \& Theureau, J. (1987). L'étude du Cours d'Action : Analyse du travail et conception ergonomique. Rapport du Laboratoire de Physiologie du travail et d'Ergonomie, Paris : CNAM.

Poizat, G., Durand, M., \& Theureau, J. (2016). The challenges of activity analysis for training objectives. Le Travail Humain. 79(3), 233-258. https://doi.org/10.3917/th.793.0233

Polya G. (1934) Patterns of plausible inférence. Princeton : Princeton University Press.

Polya, G. (1958). Les mathématiques et le raisonnement "plausible". Paris : Gauthier-Villars.

R'Kiouak, M. (2017). «Ramer ensemble » en aviron: Entre régulation inter- et extra-personnelle: Contribution à une approche enactive des couplages sociaux. Thèse de doctorat, Université de Nantes, Nantes, France.

R'Kiouak, M., Saury, J., \& Bourbousson, J. (2018). Joint action in an élite rowing pair crew after intensive team training: the reinforcement of extra-personal processes. Human Movement Science, 58, 303-313. https://doi.org/10.1016/j.humov.2017.09.008

San Martin, J. (2015). La culture d'action des enseignants de l'école primaire au Chili : Contribution au développement d'une anthropologie enactive. Thèse de doctorat, Université Toulouse II-Jean Jaurès, Toulouse, France.

Saury, J., Adé, D., Gal-Petitfaux, N., Huet, B., Sève, C., \& Trohel, J. (2013). Actions, significations et apprentissages en EPS : une approche centrée sur le cours d'expérience des élèves et des enseignants. Paris : Éditions EPS.

Seifert, L., Lardy, J., Bourbousson, J., Adé, D., Nordez, A. et al. (2017). Interpersonal coordination and individual organization combined with shared phenomenological experience in rowing performance: two case studies. Frontiers in Psychology, 8(75), https://doi.org/10.3389/fpsyg. 2017.00075

Smith, E. (2008). Social relationships and groups: new insights on embodied and distributed cognition. Cognitive systems research, 9(1-2) 24-32. https://doi.org/10.1016/j.cogsys.2007.06.011 
Sturm, S., Forster, D., \& Hutchins, E. (1997). Why Machiavelian intelligence may not be machiavelian. In A. Whiten, \& R. Byrne (Eds.), Machiavelian Intelligence II: extensions \& evaluations (pp. 50-85). New York : Cambridge University Press.

Sutton, J., Harris, C., Keil, P., \& Barnier, A. (2010). The psychology of memory, extended cognition, and socially distributed remembering. Phenomenology and the Cognitive Sciences, 9 , 521-560. https://doi.org/10.1007/s11097-010-9182-y

Terrien E., Huet B., Iachkine, P., \& Saury, J. (2020) Coordination between crew members on flying multihulls: a case study on a Nacra 17, Journal of Sport Science and Medicine, 19, 298-308. https:// jssm.org

Theureau, J. (2000). L'analyse sémio-logique des cours d'action et de leur articulation collective en situation de travail. In A. Weill-Fassina, \& T. Benchekroun (Eds.), Le travail collectif: Perspectives actuelles en ergonomie (pp. 97-118). Toulouse : Octarès.

Theureau, J. (1992/2004). Le cours d'action : Méthode élémentaire (2nd remaniée). Toulouse : Octarès.

Theureau, J. (2006). Le cours d'action: Méthode développée. Toulouse : Octarès.

Theureau, J. (2009). Le cours d'action: Méthode réfléchie. Toulouse : Octarès.

Theureau, J. (2015a). Le cours d'action : L'énaction \& l'expérience. Toulouse : Octarès.

Theureau, J. (2015b). o curso da ação. Método elementar. Belo Horizonte : Fabrefactum.

Theureau, J. (2017, Outubro). Processus créatifs : analyse de la créativité en acte. Comunicação apresentada ao Instituto de Estudos Avançados Transdisciplinares, Universidade Federal de Minas Gerais, Brasil.

Theureau, J. (2018, October). The Hutchins's heritage. Communication presented at the conference "Is Cognition Still in the Wild: A tribute to Ed Hutchins", EHESS, Paris, France.

Theureau, J. (2019). Le cours d'action : Économie \& Activités - Suivi de Note sur l'éthique. Toulouse : Octarès.

Theureau J., \& Donin, N. (2006). Comprendre une activité de composition musicale : essai méthodologique sur les relations entre sujet, activité créatrice, environnement et conscience préréflexive dans le cadre du programme de recherche « cours d'action ». In J.-M. Barbier, \& M. Durand (Eds.), Les rapports sujets-activités-environnements (pp. 221-251). Paris : PUF.

Theureau, J., \& Jeffroy, F. (Eds.) (1994). Ergonomie des situations informatisées : La conception centrée sur le cours d'action des utilisateurs. Toulouse : Octarès.

Varela, F. (2017). Le cercle créateur - Écrits (1976-2001). Paris : Seuil

Vaughan, D. (1996). The Challenger launch decision: risky technology, culture and deviance at NASA. Chicago : Chicago University Press.

Walmsley, J. (2007). Methodological situatedness: or, DEEDS worth doing and pursuing. Cognitive Systems Research, 9(1-2), 150-159. https://doi.org/10.1016/j.cogsys.2007.07.006

Watteau, B. (2017). Approche écologique de l'activité de création en formation : le cas de l'atelier de projet en architecture. Thèse de doctorat, Université Rennes, Rennes, France.

Weibel, N., Fouse, A., Emmenegger, C, Friedman, W., Hutchins, E., \& Hollan, J. (2012). Digital pen and paper practices in observational research. CHI'12: Proceedings of the SIGCHI Conference on Human Factors in Computing Systems (pp. 1331-1340). https://doi.org/10.1145/2207676.2208590 
Weibel, N., Fouse, A., Hutchins, E., \& Hollan, J. (2011). Supporting an integrated paper-digital workflow for observational research. IUI'11: Proceedings of the 16th international conference on Intelligent User Interfaces (pp. 257-266). https://doi.org/10.1145/1943403.1943443

Williams, R. (2006). Using cognitive ethnography to study instruction. ICLS'06: Proceedings of the 7th international conference on Learning sciences (pp. 838-844). https://doi.org/ $10.5555 / 1150034.1150156$

Yvon, F., \& Durand, M. (Eds.) (2012). Réconcilier recherche et formation par l'analyse de l'activité. Bruxelles : De Boeck

\section{NOTAS}

1. Pienso, por ejemplo, en la obra de Ludwig Fleck que Hutchins me hizo conocer e inversamente en la de Maurice Halbwachs (el predecesor especulativo del paradigma de la cognición distribuida) que, a cambio, le proporcioné yo mismo.

2. Como esta reflexión alcanza al conjunto del programa de investigación 'curso de acción', hubiera sido preferible que fuera colectiva desde el comienzo, y así, hubiera dado lugar a un artículo colectivo. Hasta 1990, los contactos directos entre Edwin Hutchins y sus colaboradore(as) fueron, además de Leonardo Pinsky y yo mismo, los estudiantes que preparaban su tesis de ergonomía, y también Maurice de Montmollin y Michèle Lacoste que colaboraban en ese entonces con nosotros. Luego y salvo algunas excepciones, los contactos fueron sólo conmigo, influenciando por mi intermedio al conjunto del programa de investigación 'curso de acción'. De allí surge este artículo personal destinado a dar acceso a una reflexión colectiva.

3. Agreguemos que si Edwin Hutchins acompañó la justificación de su objeto teórico, al que llama "sistema de cognición distribuida" o "ecología cognitiva", con una traducción libre (« carve nature at its joints ») de un extracto de un diálogo de Platón (Fedra, 265d-266a), si publicó ocasionalmente artículos en revistas filosóficas, yo intenté en lo personal, de manera sistemática desde 2007, precisar un programa de investigación filosófica en relación orgánica con mis investigaciones empíricas y tecnológicas, a través de varias obras (Theureau, 2009, 2015 y 2019). Pero, como la argumentación de la necesidad y la exposición del contenido de un programa de investigación filosófica ocupa demasiado espacio y como no resulta "aumentado" por la lectura de Edwin Hutchins, dejaré de lado este último.

4. Esta descripción de la sección 7 puede confundir al lector que no está familiarizado con la epistemología de los programas de investigación y con sus desarrollos. Como lo veremos inmediatamente a continuación, la noción de epistemología de programa de investigación, tal como fue definida por Imre Lakatos, incluye el cuestionamiento en todo momento por los hechos empíricos y por otros programas de investigación, de los "medios teóricos y heurísticos" del programa de investigación considerado. Una noción epistemológica de programa de investigación más desarrollada, incluye entre sus elementos, no sólo el cuestionamiento por parte de otros programas de investigación, sino también más ampliamente el conjunto de las relaciones que mantiene con otros programas de investigación

5. Es decir sin los últimos perfeccionamientos aportados a esta definición en Theureau 2015 acabamos de ver uno de ellos respecto del contenido de la sección 7- a fin de obtener las consecuencias epistemológicas de la hipótesis de la enacción. Esos últimos perfeccionamientos justifican en particular la extensión de la noción de programa de investigación, de la investigación científica, hacia la investigación tecnológica y filosófica.

6. Para la investigación sobre la actividad humana, el trabajo de Polya tiene el interés de unir el razonamiento cotidiano usual y el razonamiento matemático y científico, en particular el de Euler. 
7. Sin pretender ser exhaustivos, encontramos en la literatura internacional, dejando de lado la que se inscribe también en el programa de investigación 'curso de acción o que se inspiran en parte como Grison (1998): el "homenaje" de Bruno Latour a 'Cognition in the wild' como justificación de su propia teoría del actor-red (Latour, 1995); varias investigaciones (o mejor dicho "estudios", sin pretensiones teóricas) sobre el pilotaje de avión en relación con la seguridad aérea, sobre el pilotaje de submarino, etc., directamente inspiradas en publicaciones de Edwin Hutchins y de sus colegas; investigaciones sobre Collaborative learning (por ejemplo, Dillenbourg, Baker, Blaye, \& O'Malley, 1996); investigaciones en música sobre la Distributed creativity (Clarke, Doffman, \& Lim, 2013; Haviland, 2011; Kaastra, 2011); investigaciones sobre la memorización y el recordatorio (Sutton, Harris, Keil, \& Barnier, 2010), que remiten directamente a las antiguas especulaciones de Maurice Halbwachs sobre Les cadres sociaux de la mémoire (Halbwachs, 1925); investigaciones sobre los colectivos como sistemas dinámicos (Gorman, Dunbar, Grimm, \& Gipson, 2017). Asimismo, los cuestionamientos e intentos de superación por parte de otros investigadores sólo se refirieron al paradigma (Button, 2008; Hutto, Kirchoff, \& Myin, 2014; Smith, 2008; Walmsley, 2007). Cuando se hace referencia al método de etnografía cognitiva, como en Ball \& Ormerod (2000), Dubbels (2011) y Williams (2006), este método se reduce a la colecta de datos y a una descripción de sentido común de los fenómenos (es decir sin nociones fenomenológicas, o hasta sin sistema organizado de categorías descriptivas que remiten a hipótesis teóricas).

8. Hablaré aquí de “inflexiones", mientras que más arriba hablé de "etapas" respecto del programa de investigación 'cognición distribuida', a fin de distinguir los dos puntos de vista que rigen las descripciones efectuadas: externas, pero aprovechando interacciones productivas con Edwin Hutchins y algunos/as colaboradores/as, en lo que respecta al programa de investigación 'cognición distribuida'; internas, como consecuencia, primero, de una actividad de investigación o de intercambio de opiniones con otras investigaciones realizadas por ellos, y luego, de una actividad reflexiva que yo mismo propuse.

9. Es la primera expresión del programa de investigación 'curso de acción' (que incluye una parte importante de sus hipótesis, objetos y nociones teóricas, y de sus métodos de colecta de datos y de análisis) después de un período muy ecléctico, tanto desde el punto de vista teórico como metodológico.

10. Se pueden descargar en el sitio <Erro! A referência da hiperligação não é válida.

11. Considero aún hoy este razonamiento pragmático como válido, por lo menos mientras nuestras investigaciones se llevaban a cabo en Francia, lo que aseguraba una cultura mínima común entre los investigadores (nosotros mismos y, más generalmente, estudiantes que preparaban su tesis de ergonomía) y los actores.

12. Es interesante destacar que esta investigación fue realizada por un canadiense francófono y esencialmente en un centro donde los inspectores eran canadienses anglófonos.

13. Como empecé a hacerlo, hablaré aquí sistemáticamente de "actividades individualescolectivas y colectivas-individuales", y de "cursos de la acción individuales-colectivos y colectivos-individuales". La experiencia muestra en efecto que el adjetivo 'social' puede ser interpretado, en referencia a ciertas concepciones de lo "social", como apuntando a entidades ontológicamente diferentes de las actividades individuales-colectivas, lo que no es el caso aquí.

14. Hutchins consideraba que esos métodos producían como mucho lo que él calificaba de "discursos culturalmente correctos" (ver Theureau, 2006, p. 194-196).

15. Esos ensayos teóricos, surgidos de la insatisfacción relativa a la noción inicial de "signo tetrádico", llevaron a la noción de "signo hexádico" y a las nociones de sub-categorías de sus componentes (ver la sub-sección siguiente).

16. Por definición, una relación entre pares vincula el análisis de actividades de niveles diferentes tales como: (1) la actividad de nivel relativamente inferior pertenece a la de nivel 
relativamente superior y contribuye a su emergencia; (2) la de nivel relativamente superior fuerza a la de nivel relativamente inferior.

17. Señalo que tuve que introducir esta noción para explicar los fenómenos económicos en términos de actividades humanas

18. En lo que sigue, dejaré de lado esta última pues involucra en general a otros actores de los que se encuentran habitualmente en la situación de actividad considerada, en particular investigadores científicos y/o ingenieros y técnicos pertenecientes a otros sectores de la empresa a la que pertenecen esos actores o externos a esta última.

19. Como ejemplo elemental de tales "tendencias sintéticas de actividades", el del "efecto goteo" (trickle-down effect) (Vaughan, 1996): en una organización burocrática como es la NASA, surge que un objetivo propuesto al más alto nivel (por ejemplo, hacer que la nave espacial no sea más considerada como un objeto de investigación sino como un objeto comercial) es tomado cada vez más como una realidad a medida que se desciende en la escala jerárquica. Si el carácter burocrático de la organización constituye una limitación, el "efecto goteo" sintetiza una descripción de las actividades de los funcionarios involucrados en los diversos niveles jerárquicos.

20. Esta última es parte de dos nociones generales. La primera, empírica, es la de "mezcla conceptual" ("conceptual blend») definida como "un fenómeno cognitivo a la vez general y antiguo que consiste en la asociación de una estructura conceptual con una estructura material" y tomada de Fauconnier y Turner (2002). La "teoría de la mezcla conceptual" pretende "proporcionar un marco de análisis útil para la consideración de las contribuciones asociadas y limitaciones mutuas de las estructuras materiales. La segunda noción, a la vez empírica y tecnológica, es la de "anclaje material para una mezcla conceptual" que traduce "la proyección de la estructura material en un espacio así mezclado", proyección que "puede estabilizar la mezcla conceptual" (Hutchins, 2005, p. 1556, traducción libre). Quien conoce el programa de investigación 'curso de acción' percibe ya numerosos problemas de traducción conceptual que hay que solucionar si pensamos -me parece con razón- que es interesante inspirarse en ellos. Cuando luego Edwin Hutchins insiste en la diferencia entre lo que él llama "anclaje material" y "signo", podemos tener la impresión de encontrarnos en terreno conocido. Pero, para él, "un signo es una forma en el medio material que traduce un aspecto mínimo del campo conceptual y constituye así una muy baja forma de anclaje material" (Hutchins, 2005, p. 1573, traducción libre). En el programa de investigación "curso de acción', la noción de "signo" está concebida de manera completamente diferente y lo que está asociado a lo que se llama "anclaje" es lo que se llama "Representamen" y no lo que se llama "signo". En un caso así, nos encontramos pues realizando, no una simple integración conceptual sino una conceptualización a la vez diferente de la de partida y que enriquece o modifica a la de llegada.

21. El "observatorio", es el conjunto de los métodos de construcción de datos y las hipótesis teóricas que los acompañan, mientras que el "taller", es el conjunto de las herramientas y métodos de pre-análisis, de cálculo estadístico, de análisis y de modelización analítica y sintética y las hipótesis teóricas que los acompañan.

22. Dejaré de lado aquí la parte del taller referida a las herramientas de modelización sintética, científica y tecnológica. Esta dio lugar a diversas publicaciones, entre las cuales mencionamos por ejemplo: del lado 'cognición distribuida' (Hutchins, \& Hazlehurst, 1991, 1995); del lado 'curso de acción', (Bourbousson, \& Sève, 2010; Bourbousson, Sève, \& McGarry, 2010a, 2010b; Haradji, Guibourdenche, Reynaud, Poizat, Sabouret, Sempé, et al., 2018).

23. Mostré también en el mismo capítulo de libro que la descripción de la cognición distribuida en términos de "modelos de flujo de información" dejaba de lado fenómenos tenidos en cuenta por la descripción en términos de concatenación de signos hexádicos: los campos posibles de los actores y sus transformaciones a través de sus actividades; la selección a cada instante por cada actor de la información pertinente para él, así como los sesgos de sus interpretaciones en 
relación con su campo de posibles en ese instante; los saberes puestos en marcha y los saberes constituidos en la actividad por cada actor y su compartir colectivo más o menos efectivo; y por último, las emociones de esos actores. Una comparasión de estas mismas características fue realizada por Haué (2005) sobre la conducción automóvil.

24. La cabina (cockpit) sirvió también de banco de prueba para la plataforma de etnografía digital (Dew) y dio lugar a una nueva serie de publicaciones (ver sección 3).

25. Podríamos vernos tentados de agregar "o en un buque de guerra, situación estudiada por el programa de investigación 'cognición distribuida”, pero entonces es la ideología militar del monopolio del colectivo la que puede impedir gozar de esa facilidad.

26. Las actividades de composición musical están aún más cerca de las actividades de investigación tecnológica que de las actividades de investigación científica, como lo muestra el caso considerado en (Donin, Goldszmidt, \& Theureau, 2009) del que diré algunas palabras más adelante en esta sección, pero aquí sólo me limitaré a la investigación científica.

27. Citación original "The conduct of scientific research involves many kinds of cognitive processes: Internal processes; Processes that take place when representations are propagated across representational media; Widely distributed processes that play out in the traffic of inscriptions and the spread of ideas across scientific communities (Fleck, [1935], 1979, 2005) (...). We will address a different kind of cognitive processes that (...) occur in the interactions of scientists with one another and with material representations. They are not internal processes that accompany observed behavior; rather we will see interactions as cognitive processes" (Alac \&Hutchins, 2001, pp. 629-630).

28. Asimismo, esta extensión de la teoría de la actividad-signo a la parte de las actividades humanas que no dan lugar a conciencia prerreflexiva (o experiencia), para poder ser tratada en el marco del estudio de los cursos de in-formación y de su articulación colectiva (ver 4.3), exigiría reemplazar esta noción de conciencia prerreflexiva (o experiencia) por una noción que explique la actualidad para el actor, con la emergencia y la virtualidad que la acompañan, que englobaría esta conciencia prerreflexiva (o experiencia) y que, además, sería empíricamente tan documentable como ella, lo que plantea problemas que tampoco están resueltos.

29. Citación original "The brain has causal powers, but when it comes to human cognition, most of the causal powers of the human brain derive from the previous experience in cultural practices"(Hutchins, 2011).

\section{RESÚMENES}

El programa de investigación 'cognición distribuida', iniciado por Edwin Hutchins, ha contribuido al desarrollo del programa de investigación 'curso de acción' desde 1987 hasta nuestros días. Edwin Hutchins reconoció recientemente la proximidad de sus últimas investigaciones realizadas en términos de cognición encarnada y distribuida, con las dirigidas por el paradigma de la enacción, del que forman parte las investigaciones llevadas a cabo en el marco de este programa de investigación 'curso de acción '. Esto hace que la cuestión que se plantea hoy, sea la de definir un "programa de investigación 'curso de acción' aumentado", que, en relación con el mencionado paradigma de la enacción, por una parte, profundice y sistematice los aportes anteriores de dicho programa de 'cognición distribuida' con el programa de investigación 'curso de acción', y por otra parte, integre nuevos aportes de este último. En tal sentido, la sistematización, superación y renovación de los aportes al programa de investigación 'curso de acción' pueden apoyarse en la organización y en el cúmulo de diversos avances teóricos y metodológicos obtenidos por ambos 
programas de investigación. Vamos a ver aquí qué respuesta podemos aportar personalmente a esta cuestión.

O programa de pesquisa "cognição distribuída", iniciado por Edwin Hutchins, contribuiu para o desenvolvimento do programa de pesquisa "curso de ação" de 1987 até aos dias atuais. Edwin Hutchins reconheceu recentemente a proximidade das suas últimas investigações, realizadas em termos de cognição corporificada e distribuída, com aquela regida pelo paradigma da enação, cuja investigação é desenvolvida no âmbito deste programa de investigação " curso de ação". Coloca-se agora a questão da definição de um "programa de pesquisa de "curso de ação" ampliado", que, em relação a este paradigma de atuação, por um lado, aprofunda e sistematiza as contribuições anteriores deste programa de pesquisa de "cognição distribuída" para o programa de pesquisa de "curso de ação", e, por outro lado, integra novas contribuições deste último. Tal sistematização, superação e renovação das contribuições para o programa de pesquisa do "curso de ação" podem basear-se na mutualização e acumulação de vários avanços teóricos e metodológicos obtidos pelos dois programas de pesquisa. Veremos aqui que resposta podemos fornecer pessoalmente a esta pergunta.

Le programme de recherche " cognition distribuée », initié par Edwin Hutchins, a contribué au développement du programme de recherche "cours d'action» de 1987 à nos jours. Edwin Hutchins ayant reconnu récemment la proximité de ses dernières recherches, menées en termes de cognition incarnée et distribuée, avec celles qui sont commandées par le paradigme de l'énaction, dont font partie les recherches menées dans le cadre de ce programme de recherche "cours d'action", la question se pose aujourd'hui de la définition d'un "programme de recherche "cours d'action" augmenté », qui, en relation avec ce paradigme de l'énaction, d'une part, approfondisse et systématise les apports antérieurs de ce programme de recherche « cognition distribuée » au programme de recherche "cours d'action », d'autre part, intègre de nouveaux apports de celui-ci. Une telle systématisation, un tel dépassement et un tel renouvellement des apports au programme de recherche "cours d'action » peuvent s'appuyer sur la mutualisation et le cumul de divers progrès théoriques et méthodologiques obtenus par les deux programmes de recherche. Nous allons voir ici quelle réponse nous pouvons apporter personnellement à cette question.

The 'distributed cognition' research program, initiated by Edwin Hutchins, has contributed to the development of the 'course of action' research program from 1987 until now. Edwin Hutchins has recently recognized the proximity between his last researches, developed in terms of embodied and distributed cognition, and the enaction paradigm, followed by this 'course of action' research program. The question of the definition of an "augmented 'course of action' research program" is now raised. Such "program" should, in relation with this enaction paradigm, deepen and synthetize the past contributions of the 'distributed cognition' research program to the 'course of action' research program and add new contributions from it. Such deepening, synthesis, and renewal of the contributions to the 'course of action' research program are made easy by the mutual contribution and addition of different theoretical and methodological results obtained by the two research programs. We will present here our personal answer to this question. 
ÍNDICE

Keywords: distributed cognition, course of action, cognitive ethnography, cultural practice, mutual appropriation

Palavras-chave: cognição distribuída, curso de ação, etnografia cognitiva, prática cultural, apropriação mútua

Palabras claves: cognición distribuida, curso de acción, etnografía cognitiva, práctica cultural, Apropiación mutua

Mots-clés: cognition distribuée, cours d'action, ethnographie cognitive, pratique culturelle, appropriation mutuelle

\section{AUTOR}

\section{JACQUES THEUREAU}

Investigador (en retiro) Institut de Recherche-Coordination Acoustique-Musique (IRCAM)

1 place Igor Stravinsky, 75004, Paris

jacques.theureau@ircam.fr 